\title{
Secretome Analysis and In Planta Expression of Salivary Proteins Identify Candidate Effectors from the Brown Planthopper Nilaparvata lugens
}

\author{
Weiwei Rao, Xiaohong Zheng, Bingfang Liu, Qin Guo, Jianping Guo, Yan Wu, Xinxin Shangguan, \\ Huiying Wang, Di Wu, Zhizheng Wang, Liang Hu, Chunxue Xu, Weihua Jiang, Jin Huang, Shaojie Shi, \\ and Guangcun $\mathrm{He}^{\dagger}$
}

National Key Laboratory of Hybrid Rice, College of Life Sciences, Wuhan University, Wuhan, Hubei, China

Accepted 30 July 2018.

\begin{abstract}
The brown planthopper (BPH), Nilaparvata lugens (Stål), is a phloem sap-feeding insect. During feeding on rice plants, BPH secretes salivary proteins with potential effector functions, which may play a critical role in the plant-insect interactions. However, a limited number of BPH effector proteins have been identified to date. Here, we sequenced the salivary gland transcriptomes of five BPH populations and subsequently established a $N$. lugens secretome consisting of 1,140 proteinencoding genes. Secretome analysis revealed the presence of both conserved and rapidly evolving salivary proteins. A screen for potential effectors that elicit responses in the plant was performed via the transient expression analysis of $64 \mathrm{BPH}$ salivary proteins in Nicotiana benthamiana leaves and rice protoplasts. The salivary proteins NI12, Nl16, Nl28, and NI43 induced cell death, whereas NI40 induced chlorosis and NI32 induced a dwarf phenotype in $N$. benthamiana, indicating effector properties of these proteins. Ectopic expression of the six salivary proteins in $N$. benthamiana upregulated expression of defense-related genes and callose deposition. Tissue expression analysis showed a higher expression level of the six candidate effectors in salivary glands than in other tissues. Subcellular localization and analysis of the domain required for cell death showed a diverse structure of the six effectors. N128, N140, and N143 are $N$. lugens specific; in contrast, NI12, NI16, and NI32 are conserved among insects. The N140 family has numerous isoforms produced by alternative splicing, exemplifying rapid evolution and expansion of effector proteins in the BPH. Our results suggest a potential large effector repertoire in $B P H$ and a higher level of effector conservation exist in BPH compared with that in plant pathogens.
\end{abstract}

Nilaparvata lugens (Stål), commonly known as the brown planthopper (BPH), is a major rice pest that has coevolved with

W. Rao and X. Zheng contributed equally to this work.

${ }^{\dagger}$ Corresponding author: Guangcun He; E-mail: gche@whu.edu.cn

Funding: This work was supported by the National Natural Science Foundation of China (grant numbers 31230060 and 31171525 ).

*The $\boldsymbol{e}$-Xtra logo stands for "electronic extra" and indicates that one set of supplementary data, eight supplementary figures, and eight supplementary tables are published online.

๑) 2019 The American Phytopathological Society rice for approximately 0.25 million years (Sezer and Butlin 1998). Serious damage of BPH to rice can result in "hopper burn". During feeding on rice, BPH use their stylets to puncture the plant epidermis, followed by probing and penetration of the plant cell wall to access the phloem sap (Wang et al. 2008). During this process, BPH secretes both gelling and watery saliva from their salivary glands into plant cells. Gelling saliva quickly solidifies following secretion and forms a continuous salivary sheath in the plant that provides mechanical stability, lubrication, and protection against plant defense chemicals for the insect, whereas watery saliva contains digestive, hydrolyzing, and cell-wall-degrading enzymes (Miles 1999). The saliva of herbivorous insects plays a crucial role in their interaction with plants, and determines the compatibility between feeding insects and host plants (Miles 1999). Broadly, salivary proteins and other molecules secreted into plant cells by insects that can alter host structures and functions are defined as effectors (Hogenhout et al. 2009). Many sap-feeding insects cause extensive losses in modern-day agriculture. Only recently has the characterization of effectors from sap-feeding insects been a research focus, and studies have primarily focused on aphids and the Hessian fly. Several insect effectors have been identified in the past few years (Jaouannet et al. 2014; Kaloshian and Walling 2016; Mutti et al. 2008; Naessens et al. 2015; Rodriguez et al. 2014; Wang et al. 2015a; Zhang et al. 2015), providing insights into the mechanisms underlying plant-insect interactions.

To overcome plant defense responses, plant parasites such as bacteria, fungi, oomycetes, nematodes, and insects secrete a repertoire of effectors into plant cells. In total, 758 secreted proteins were predicted in the genome of the fungal pathogen Ascochyta rabiei. Secretome analysis identified several secreted proteins with numerous tandem repeats, which provides insights into the necrotrophic nature of fungal phytopathogens (Verma et al. 2016). A bioinformatics approach identified 486 secreted proteins in the fungal pathogen Sclerotinia sclerotiorum, and further analysis of the secretome revealed 78 secreted proteins associated with positive selection, recent gene duplication, S. sclerotiorum-specific effectors, and effector candidates associated with broad-host-range necrotrophy (Guyon et al. 2014). In insects, a dual transcriptomic and proteomic approach identified 324 secreted proteins in the aphid Acyrthosiphon pisum. Furthermore, it was demonstrated that multiple-copy genes in A. pisum genome are under positive selection and many secreted proteins appear to evolve at a faster rate compared with that of proteins homologous to those in other insects (Carolan et al. 2011). 
Many pathogen effectors may evolve rapidly, thus exhibiting a high level of sequence polymorphism and species specificity (Guyon et al. 2014; Raffaele et al. 2010). However, insects, as multicellular eukaryotes at an evolutionary level much higher than that of bacteria, fungi, and oomycetes, have a lower evolutionary rate than the latter. Several effector genes identified in insects display high-level sequence conservation among different species, despite their different function in their respective host plants. The first salivary protein characterized to suppress host defenses was glucose oxidase (GOX) (Musser et al. 2002), which is conserved among many caterpillar species and sapfeeding insects such as aphids (Eichenseer et al. 2010; Harmel et al. 2008). A comparative analysis of the salivary proteins in blood- and plant-feeding insects revealed a number of common types such as apyrases, calreticulins, and peroxiredoxins that interfere with early host defense signaling (Guiguet et al. 2016). Recent studies have identified several conserved effectors in aphids. Many vertebrate parasites, including protozoa, nematodes, and ticks, secrete macrophage migration inhibitory factor (MIF) to modulate host defense responses (Augustijn et al. 2007; Bowen et al. 2010; Vermeire et al. 2008). MIF secreted by a plant-parasitic aphid inhibits plant defense responses and is crucial for the survival and feeding of the aphid on the host plant (Naessens et al. 2015). Armet, a conserved protein in many animal species, has been detected in aphid watery saliva and mediates aphid-plant interactions. Armet facilitates pea aphid feeding on bean plants (Wang et al. 2015a). Angiotensinconverting enzymes (ACEs), which are conserved in animals, are highly expressed in the $A$. pisum salivary glands. Simultaneous knockdown of $A C E 1$ and ACE2 confers a higher mortality rate than the control group in aphids feeding on plants, indicating that ACE1 and ACE2 function together to modulate aphid-plant interactions (Wang et al. 2015b). Mp10, a chemosensory protein (CSP) in the aphid Myzus persicae, induces chlorosis and triggers defense responses in Nicotiana benthamiana (Bos et al. 2010; Rodriguez et al. 2014). Furthermore, the CSP family displays a notable level of conservation in insects.

Past studies have reported the identification of either 202 or $107 \mathrm{BPH}$ salivary proteins via mass spectrometric analysis of saliva collected in vitro (Huang et al. 2016; Liu et al. 2016). However, only a few BPH effectors have been identified (Huang et al. 2017; Ji et al. 2017; Shangguan et al. 2018; Ye et al. 2017). Pathogen recognition in plants is often accompanied by the hypersensitive response, a form of programmed cell death. Transient expression of secreted proteins in $N$. benthamiana combined with cell death assays has been developed as a convenient tool to identify candidate pathogen effectors (Chen et al. 2018; Kettles et al. 2017; Wang et al. 2011). In this study, we characterized the BPH secretome containing 1,140 potential secreted proteins. In total, 64 salivary proteins were analyzed using $N$. benthamiana cell death assays. Six were identified as candidate effector proteins and subsequently applied to function analysis. Particular attention was paid to the differences between insect effectors and pathogen effectors, including subcellular localization, sequence, and structure features. Our results indicate that the effector repertoire in $\mathrm{BPH}$ is large and that many $\mathrm{BPH}$ effectors are conserved proteins.

\section{RESULTS}

\section{Sequencing of the BPH salivary gland transcriptome and prediction of secreted proteins.}

The cDNA libraries produced using the salivary glands of five BPH populations, which were reared on either plants of the rice varieties TN1 (TN1 population), Mudgo (M population),
ASD7 (A population), and YHY15 (Y population) or Leersia (L population) plants, were sequenced using an Illumina platform, which generated a total of 40,001,367, 10,0591,728, 100,249,608, 40,000,103, and 98,534,746 reads, respectively. Sequences were assembled using SOAPdenovo software ( $\mathrm{Li}$ et al. 2009), resulting in 31,645, 34,245, 25,535, 41,676, and 29,119 unigenes, respectively. Finally, sequence data from the five libraries were combined and assembled into 41,883 unigenes. All the raw transcriptome sequencing data were deposited in the SRA database (National Center for Biotechnology Information [NCBI]) under accession numbers SRX532392 (TN1 population), SRX2882231 (M population), SRX2882276 (A population), SRX2882277 (Y population), and SRX2882278 (L population).

Secreted proteins are likely deposited in the saliva through the eukaryotic endoplasmic reticulum-Golgi pathway (Cherqui and Tjallingii 2000). Therefore, all amino acid sequences were assigned to predict secretory signal peptides using SignalP 4.1 (Petersen et al. 2011), and 1,315 unigenes were predicted to encode proteins containing a secretory signal peptide. Finally, following the removal of 175 unigenes containing a transmembrane domain in addition to the signal peptide, 1,140 potential secreted proteins were isolated, forming a Nilaparvata lugens secretome (Supplementary Table S1). The size of the $N$. lugens secretome reported here is considerably larger than that previously predicted ( $\mathrm{i}$ et al. 2013), and resembles the numbers of secreted proteins reported for the spider mites Tetranychus urticae $(1,493)$ and T. evansi $(1,121)$ (Villarroel et al. 2016).

Past studies have reported $83 \mathrm{~N}$. lugens salivary proteins that contain a signal peptide by mass spectrometry (Huang et al. 2016; Liu et al. 2016). Following the removal of redundant proteins, we obtained a dataset of 65 salivary proteins with signal peptide. We used these 65 salivary proteins to BLAST against the $N$. lugens secretome $(1,140)$ with a cut-off E-value of $10^{-5}$, which resulted in 61 sequence matches, indicating that this secretome is of high quality.

\section{Characterization of the BPH secretome.}

Rapid effector evolution and extensive expansion of specific effector families has been demonstrated in the genome of the oomycete Phytophthora infestans (Haas et al. 2009). Therefore, we clustered proteins identified in the $\mathrm{BPH}$ secretome into protein families using orthoMCL ( $\mathrm{Li}$ et al. 2003). In total, 746 proteins were clustered into 174 protein families with more than 2 proteins (Supplementary Table S2). The largest protein family consisted of 25 proteins without annotation. Annexin proteins, previously identified in the saliva of BPH (Huang et al. 2016), constituted the second-largest protein family. Furthermore, particular attention was paid to large protein families which have highly expressed members. With the application of this condition, groups 3, 6, 19,31, 70, and 97 of the 174 protein families were selected for further investigation.

Insects share a number of salivary proteins that interfere with early host defense signaling (Guiguet et al. 2016). To identify insect-shared salivary proteins in BPH, we used the $N$. lugens secretome $(1,140$ proteins) to search the now-published secretome of two insect species, T. urticae secretome (1,493 proteins) (Villarroel et al. 2016) and A. pisum secretome (324 proteins) (Bos et al. 2010; Carolan et al. 2011), using the BLAST tool with an E-value of $10^{-5}$, which obtained 245 and 229 homologous proteins, respectively (Supplementary Table S3). In order to identify $N$. lugens-specific salivary proteins, we used the $N$. lugens secretome to search the $\mathrm{Nr}$, Swiss-Prot database and the transcriptomes of six insect species (A. pisum, Tribolium castaneum, Drosophila melanogaster, Nasonia vitripennis, Manduca sexta, and Locusta migratoria) representing 
six different insect orders. As a result, 461 proteins $(40.44 \%)$ could not match proteins of other species in these databases and were considered to be Nilaparvata lugens-specific proteins, and the rest $679(59.56 \%)$ were regarded as conserved proteins.

BPH secretes both watery and gelling saliva, and the latter solidifies to form a feeding sheath. Recently, 10 (NCBI accession numbers BAP87097.1 to BAP87106.1) and 13 (Huang et al. 2016) gelling saliva proteins with signal peptides from $\mathrm{BPH}$ were identified. After removing repetitive proteins, 20 gelling saliva proteins were finally obtained (Supplementary Table S4). Among these, 19 were identified in our N. lugens secretome. Comparative analysis showed that 30 of the top 50 highly expressed genes in our secretome are gelling saliva proteins, indicating that many salivary sheath proteins are highly expressed in salivary glands. Furthermore, 13 of the 20 feeding sheath proteins shared no similarity with proteins from other organisms contained in the NCBI database.

Amino acid tandem repeats exist in many eukaryotic proteins (Huntley and Golding 2000). Although the function of amino acid tandem repeats remains unclear, natural selection drives the accumulation of amino acid tandem repeats by modulating repeat size and number (Mularoni et al. 2010). Amino acid tandem repeats were identified in the $N$. lugens secretome, with 254 proteins $(22.24 \%)$ containing at least two amino acid tandem repeats of two residues or more (Supplementary Table S5). The presence of amino acid tandem repeats was much higher in the highly expressed proteins than in the entire secretome. Among the 20 highly expressed salivary sheath proteins, 9 contain amino acid tandem repeats, typically are mucin-like protein (accession number BAP87097.1) and vitellogenin (accession number BAP87098.1), in which tandem repeat sequences are abundant. Furthermore, when the secreted proteins were ranked according to gene expression level (T_FPKM), 42 genes in the top 100 highly expressed genes were found to contain amino acid tandem repeats. This result suggests the rapid evolution of the highly expressed salivary proteins in N. lugens.

Most fungal effector proteins identified to date are small secreted cysteine-rich proteins (SSCPs), which are small in size $(<200$ amino acids [aa]) and have a high cysteine content $(>2 \%)$ (Saunders et al. 2012; Stergiopoulos and de Wit 2009). The cysteines in SSCPs are often involved in the formation of disulfide bonds, which stabilize protein structures (Saunders et al. 2012). SSCPs are recognized as a common source of fungal effectors that trigger defense responses in host plants. Thus, we characterized the cysteine content of all $N$. lugens secretome proteins and identified 432 (37.89\%) cysteine-rich proteins for which $2 \%$ or more of amino acid residues are cysteine.

\section{Identification of BPH candidate effectors.}

Previous studies have shown that many pathogen effector proteins can induce cell death in nonhost plants (Wroblewski et al. 2009). To identify candidate BPH effectors, we used transient expression of $\mathrm{BPH}$ salivary proteins in Nicotiana benthamiana via agroinfiltration to screen for those salivary proteins that induce cell death.

Candidate effectors were identified in the Nilaparvata lugens secretome using selection criteria: high expression level in salivary glands, member of a large protein family (i.e., 13 genes from groups 3, 6, 19, 31, 70, and 97 were selected), homolog of known effectors in other organisms, and $N$. lugens-specific genes. The cDNAs of 64 salivary proteins were successfully cloned from BPH biotype I (Supplementary Table S6). Because salivary proteins may be secreted in both the plant cell and apoplast, for each of these genes, two plasmids were constructed: one contained an open reading frame with signal

peptide $(\mathrm{ORF}+\mathrm{SP})$ and the other contained a truncated open reading frame without signal peptide (ORF-SP).

Transient expression assays showed that 6 of the 64 salivary proteins (namely, N112, N116, N128, N132, N140, and N143) induced cell death, chlorosis, or a dwarf phenotype in Nicotiana benthamiana (Fig. 1A), indicating that these proteins may function as effectors that are recognized by the plant immune system (Wang et al. 2011). N112, N116, and N128 induced cell death in $N$. benthamiana only when expressed without a signal peptide (Fig. 1A). In contrast, N140 induced chlorosis and N143 induced cell death in $N$. benthamiana when expressed with a signal peptide (Fig. 1A). Interestingly, N132 expressed both with and without a signal peptide induced a dwarf phenotype in $N$. benthamiana (Fig. 1A). Immunoblot analysis confirmed the expression of these proteins in $N$. benthamiana (Supplementary Fig. S1).

To further confirm candidate BPH effector functionality, the six proteins described above were coexpressed with the luciferase reporter protein in rice protoplasts to determine whether they induced cell death in rice (Chen et al. 2006). Compared with that in the green fluorescent protein (GFP) control, expression of N112, N128, and N143 caused a significant reduction in luciferase activity whereas N116 caused a slight reduction

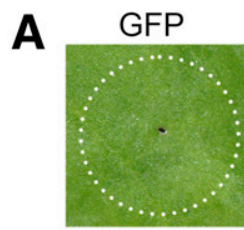

NI40

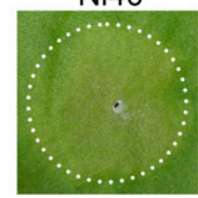

B

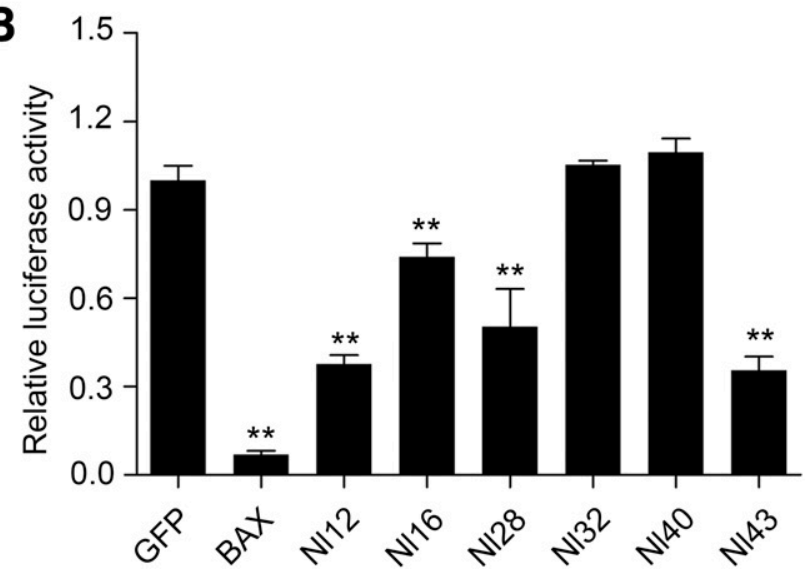

Fig. 1. Brown planthopper (BPH) candidate effectors induce cell death in Nicotiana benthamiana and rice protoplasts. A, Analysis of BPH effector functionality in $N$. benthamiana. Six candidate BPH effectors were transiently expressed in $N$. benthamiana leaves by agroinfiltration with PVX vectors alongside green fluorescent protein (GFP) that was included as a control. Pathology symptoms were documented with photos taken 7 days postinfiltration (DPI) for N112, N116, N128, N140, and N143 and 15 DPI for N132. B, Analysis of BPH effector functionality in rice protoplasts. Six candidate BPH effectors and GFP were coexpressed with the luciferase reporter protein in rice protoplasts. Values are mean $(n=3)$ relative luciferase activity in tissue expressing a candidate effector normalized against tissue expressing GFP, the latter of which was set to one. Cell death induced by candidate effectors was reflected by a reduction in relative luciferase activity. Error bars show \pm standard deviation and asterisks $(* *)$ significant difference, $P<0.01$ (Student's $t$ test). Luciferase activity was measured using a luciferase assay system (Promega Corp.) $40 \mathrm{~h}$ after transformation. 
(Fig. 1B), confirming that these proteins induce cell death in rice protoplasts. Transient expression of N132 and N140 did not affect luciferase activity (Fig. 1B).

\section{Tissue expression profiles of BPH candidate effectors.}

Some salivary proteins of BPH may be exclusively expressed in the salivary glands. To investigate the expression patterns of the six candidate effectors in BPH, different tissues, including salivary glands, midguts, fat bodies, and the remaining parts, were dissected from BPH adults and were used for real-time quantitative polymerase chain reaction (RT-qPCR) analysis. Nl12 and N116 were mainly expressed in the salivary glands but low expression levels were also detected in other tissues (Fig. $2 \mathrm{~A}$ and B). N128 was detected at high expression levels in both the salivary glands and midguts (Fig. 2C). As for N132, transcripts were extensively detected in all test tissues but were highest in the salivary glands (Fig. 2D). N140 and N143 were exclusively expressed in the salivary glands and almost undetectable in other tissues (Fig. 2E and F).

\section{BPH candidate effectors activate plant defense responses.}

To analyze whether the six cell death-inducing proteins N112, Nl16, Nl28, Nl32, Nl40, and N143 affected the plant immune system, we transiently expressed these proteins in $N$. benthamiana leaves and then analyzed defense-related gene expression and callose deposition.

The relative expression of the salicylic acid (SA)-related marker genes pathogenesis related $1(P R I)$ and $P R 2$ and the jasmonic acid (JA)-related marker genes $P R 3$ and $P R 4$ were determined using RT-PCR analysis performed 2 days postinfiltration (DPI) (Keller et al. 1996; Maimbo et al. 2010).
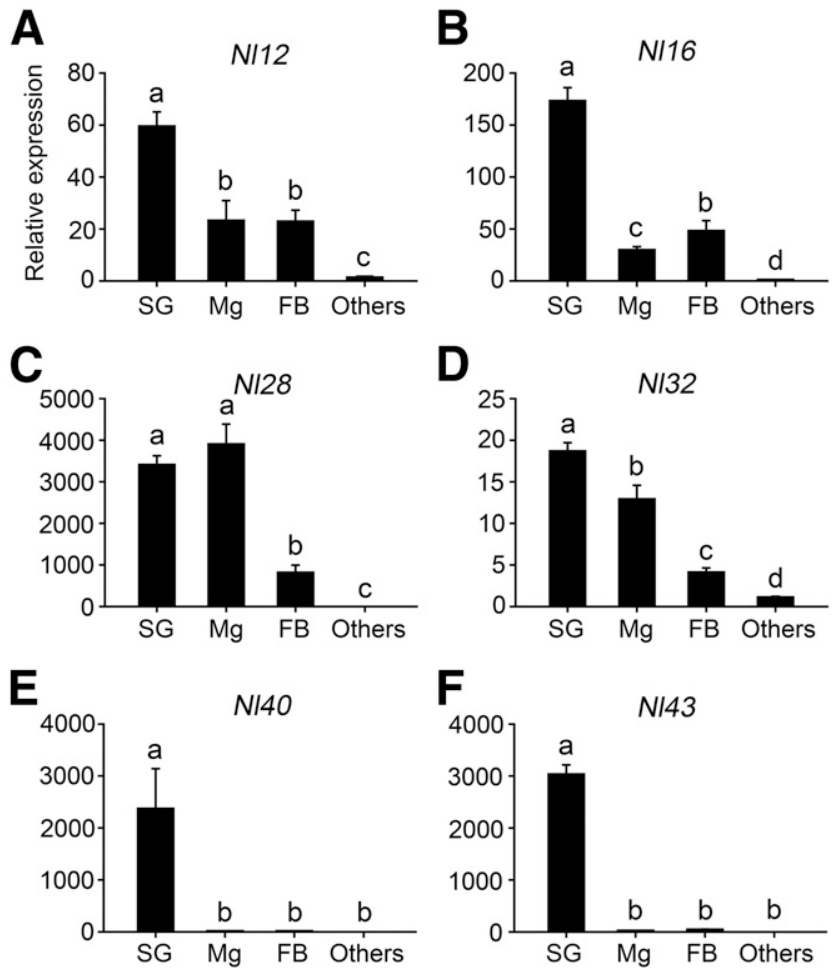

Fig. 2. Expression analysis of brown planthopper (BPH) candidate effectors. Relative expression levels of BPH candidate effectors in different tissues were normalized against $\beta$-actin gene expression. The remaining parts dissected from BPH was used as reference. Error bars denote \pm standard deviation of three repeats. A, Nl12; B, Nl16; C, Nl28; D, Nl32; E, Nl40; and F, Nl43. Different letters above the bars indicate significant differences, $P<0.05$ (Tukey's honestly significant difference test). SG = salivary glands, $\mathrm{Mg}=$ midguts, $\mathrm{FB}=$ fat bodies, and Others $=$ the remaining parts.
Surprisingly, we found that expression of N112 induced approximately 9- and 59-fold expression upregulation of the JArelated marker genes $P R 3$ and $P R 4$, respectively, and N116 expression induced approximately 16 -fold expression upregulation of SA-related marker genes $P R 1$ and $P R 2$ (Fig. 3A). Moreover each SA- and JA-related marker gene displayed significant expression upregulation following N132 expression whereas N128 caused significant and simultaneous expression upregulation of $P R 1, P R 2$, and $P R 4$ (Fig. 3A). In contrast, the remaining BPH candidate effectors $\mathrm{Nl} 40$ and N143 caused a slight reduction of both SA- and JA-related marker genes expression (Fig. 3A).

Callose deposition is a hallmark of plant defense responses and is typically triggered by pathogen-associated molecular patterns (PAMPs) (Luna et al. 2011). Aside from PAMPs, pathogen effectors and injury caused by insects can trigger callose depositions (Kuśnierczyk et al. 2008; Naessens et al. 2015; Voigt 2014). Callose deposition assays were performed in $N$. benthamiana leaves, as described previously (Dietrich et al. 1994; Naessens et al. 2015). We observed that N116, N128, N132, and N140 expression caused enhanced callose deposition at 2 DPI (Fig. 3B). Furthermore, rapid and strong callose deposition was detected at 2 DPI for N112 and N143 (Fig. 3B).

\section{Subcellular localization of BPH candidate effectors in plant cells.}

During feeding on rice, $\mathrm{BPH}$ penetrates the plant tissue with the stylets and delivers salivary proteins into plant cells (Wang et al. 2008). Identifying the subcellular localization of effector proteins inside the plant cell can provide information about their mode of action (Dowen et al. 2009; Schornack et al. 2010).

To determine the subcellular localization of the six BPH candidate effectors in the plant cell, the signal peptide in each of the six proteins was removed; then, C-terminal fusion proteins with the yellow fluorescent protein (YFP) were created and expressed in rice protoplasts. A strong fluorescence signal for N112-YFP, N116-YFP, and Nl28-YFP was observed in both the cytoplasm and the nucleus (Fig. 4). N132-YFP was primarily localized in the nucleus, with slightly fluorescence signal observed also in the cytoplasm (Fig. 4). The observed localization pattern of N132 differed from that of the homolog effector Mp10 in Myzus persicae, which is localized in the cytoplasm when expressed in $N$. benthamiana (Rodriguez et al. 2014). Fluorescence observed for N140-YFP and N143-YFP indicated that they were both localized in the nucleus (Fig. 4).

We also investigated the subcellular localization of the six BPH candidate effector-YFP fusion proteins in N. benthamiana, with results similar to those observed in rice protoplasts, except that N140-YFP was both nuclear and chloroplast localized in $N$. benthamiana and $\mathrm{N1} 32$ was only localized in the nucleus (Supplementary Fig. S2). From the above results, we concluded that the six BPH candidate effectors (N112, Nl16, Nl28, Nl32, N140, and N143) localized in the nucleus or nucleus and cytoplasm, similar to the subcellular localization pattern of oomycete RXLR (a conserved motif in oomycete effectors) effectors (Caillaud et al. 2012). However, in silico analysis showed that none of the six proteins carry a canonical nuclear localization signal. Western blot using GFP antibodies showed the expression of the effector-YFP fusion proteins (Supplementary Fig. S3).

\section{Structural analysis of BPH candidate effectors required for cell death.}

The structure of protein is closely related to its function. To identify the functional protein domains in the BPH candidate effectors required to induce plant responses, a series of effector deletion mutants were constructed and analyzed via cell death assays. 
N112 is a protein disulfide isomerase (PDI) and consists of the four thioredoxin-like domains $\mathrm{a}, \mathrm{a}^{\prime}, \mathrm{b}$, and $\mathrm{b}^{\prime}$; an N-terminal signal peptide; and a C-terminal endoplasmic reticulum (ER) retention signal (Fig. 5A) (Ben Achour et al. 2002; Meng et al. 2015). Cell death assay results showed that N112 induced cell death only when the signal peptide was truncated, indicating a secretion function of this protein in plant cells when it had a signal peptide and an ER retention signal simultaneously, whereas the ER retention signal KDEL was not required for N112-induced plant symptoms (Fig. 5A). A minimum of three PDI domains were required for N112 function, and the engineered versions of Nl12 containing domain combinations a-b$\mathrm{b}^{\prime}$ or $\mathrm{b}-\mathrm{b}^{\prime}-\mathrm{a}^{\prime}$ were able to induce cell death in $N$. benthamiana (Fig. 5A). Thus, it appears that the domains a and $\mathrm{a}^{\prime}$ share a similar role in N112 function.

N116 is an apolipophorin-III composed of a signal peptide and an ApoLp-III_like domain that spans the majority of the protein (30 to 179 aa, 79\%). The ApoLp-III_like domain alone was sufficient for N116 function in N. benthamiana (Fig. 5B). Furthermore, the minimal functional sequence within the ApoLpIII_like domain was identified as 61 to 140 aa, which induced more extensive cell death compared with that resulting from fulllength N116 without its signal peptide (N116-SP) (Fig. 5B).

N128 is an SSCP. N128 induced weak symptoms in N. benthamiana (Fig. 1A). However, cell death was significantly enhanced following the expression of N128 with a C-terminal hemagglutinin (HA) tag fusion. Therefore, we used the N128$\mathrm{HA}$ in further experiments. The N128 protein sequence contains no predicted domains, and therefore we created successive protein truncations reducing the amino acid sequence by 50 aa, 30 aa, and 10 aa. This narrowed the N128 functional region to 101 to 167 aa located in the C-terminus (Fig. 5C).

$\mathrm{N} 132$ is a CSP that contains an OS-D domain (insect pheromone-binding family) located in the middle of the amino acid sequence, which spans a large proportion of the protein (28 to 118 aa, approximately $70 \%$ ). Compared with the a dwarf phenotype induced by N132 (Fig. 1A), the OS-D domain C- terminal truncated form (28 to 111 aa) had much weaker effects, and the OS-D domain N-terminal truncated form (38 to 118 aa) did not induce a plant response, indicating that the complete OS-D domain is required for $\mathrm{Nl32}$ function in N. benthamiana (Fig. 5D).

N143 induced extensive cell death in $N$. benthamiana when expressed with its endogenous signal peptide (Fig. 5E). The N143 protein sequence contains no predicted domains and, therefore, we divided the protein into an $\mathrm{N}$-terminal and a Cterminal segment (1 to 140 and 141 to 244 aa, respectively), each with a signal peptide. Functional assessment of sequential 20 -aa protein truncations showed that the minimal N143 region required for the induction of cell death was the C-terminal 141 to 244 aa (Fig. 5E). We did not carry out the "deletion mutant" experiments for N140, because chlorosis induced by N140 is too weak and cannot induce cell death in rice protoplast.

\section{Sequence analysis and homology search}

of the BPH candidate effectors.

Sequence analysis and homologs of the BPH candidate effectors in other organisms may provide some insight into the function of these proteins.

Nl12. N112 is a member of the PDI family, which is conserved in eukaryotic organisms. Through reciprocal BLAST analysis, 13 NIPDI family members were identified in the BPH transcriptome and 5 PDI family members were identified in the Nilaparvata lugens secretome (Supplementary Table S7). Nl12 shares 37, 56, and 57\% similarity with gene homologs in P. parasitica (PpPDI1), Oryza sativa, and Nicotiana benthamiana, respectively. Sequence alignment showed that PDIs in insects, oomycetes, fungi, and plants were highly conserved. (Supplementary Fig. S4).

Nl16. N116 is an apolipophorin-III protein. Apolipophorin-III is a canonical apolipoprotein with an ApoLp-III_like domain, which is conserved in many insect species (Supplementary Fig. S5). Homology searches in our transcriptome revealed no homologous gene for Nl16.
A

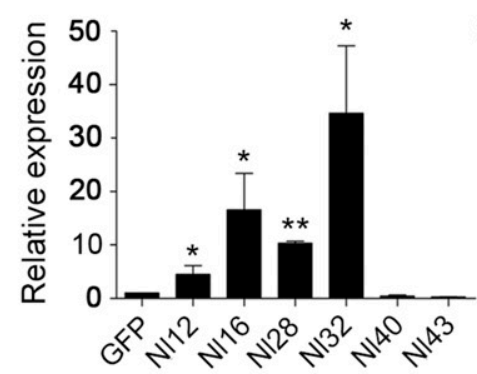

B GFP

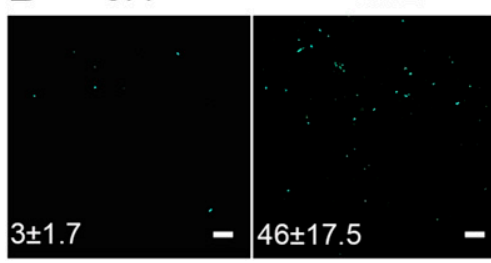

$\mathrm{NbPR2}$

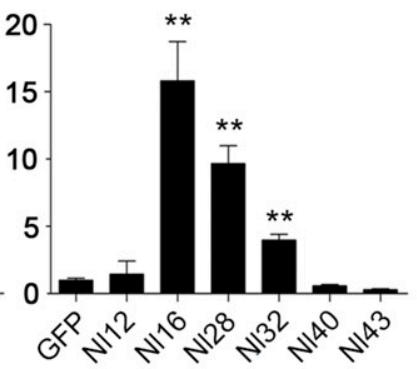

$\mathrm{NbPR3}$

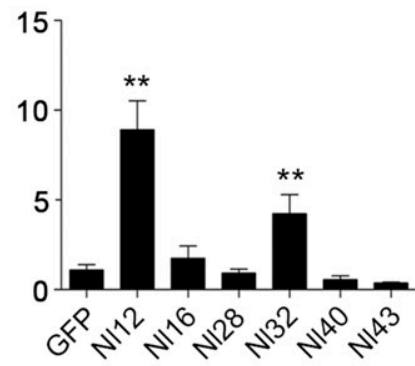

$\mathrm{NbPR4}$

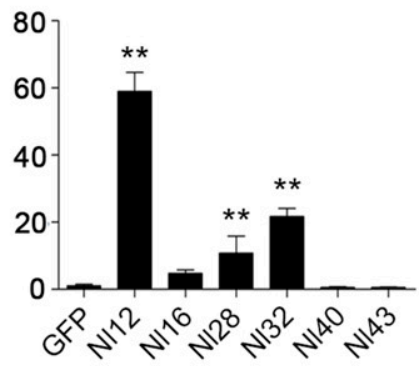

Fig. 3. Brown planthopper (BPH) candidate effectors activate plant defense responses. A, BPH candidate effectors affect the expression of defense-related genes in Nicotiana benthamiana. Following the transient expression of six candidate BPH effectors in N. benthamiana, relative expression of the salicylic acidand jasmonic acid-related marker genes pathogenesis-related (PR) genes $P R 1 / P R 2$ and $P R 3 / P R 4$, respectively, were measured by real-time polymerase chain reaction at 2 days postinfiltration. Values are mean $(n=3)$ relative expression in tissue expressing a candidate effector normalized against tissue expressing green fluorescent protein (GFP), the latter of which was set to one. Error bars denote \pm standard deviation and $* *$ indicates significant difference, $P<0.01$ (Student's $t$ test). B, BPH candidate effectors induce callose deposition in $N$. benthamiana leaves. Callose deposition was detected in detached leaves stained with aniline blue. GFP (left panel) was infiltrated as a control. Numbers indicate \pm standard deviation of mean values $(n=3)$. For each experiment, callose deposition was quantified in a minimum of 10 randomly selected microscopy fields using ImageJ software. Scale bars $=100 \mu \mathrm{m}$. 
Nl28. N128 is an SSCP for which no homologous gene was identified in the NCBI database and BPH transcriptome. N128 (N128-1) has a particularly high cysteine content of $34 \%$. Therefore, we selected an additional four SSCPs with a cysteine content higher than $15 \%$ (Supplementary Fig. S6) and observed cell-death symptoms following their transient expression in $N$. benthamiana, which revealed that three of these SSCPs induced a weak cell death.

Nl32. CSPs, a family of small water-soluble proteins with an OS-D domain, are conserved among different insects (Pelosi et al. 2005). Eleven CSPs (NlCSP1 to -11) have been previously identified in BPH (Zhou et al. 2015). We identified five CSPs (namely, NlCSP1 [N132], N1CSP5, NlCSP6, NlCSP7, and NICSP8) in the Nilaparvata lugens secretome. Of these, NICSP1 (N132) and NICSP5 were highly expressed in salivary glands.

Nl40. N140 is a N. lugens-specific salivary protein which has no homolog in other organisms. N140 shares $80 \%$ similarity with a salivary protein (accession number KU365932) detected in both the watery and gelling saliva of BPH (Huang et al. 2016). Nl40 was categorized into group 6 alongside 14 unigenes in our $N$. lugens secretome. To investigate the level of $N l 40$ polymorphism in BPH, 100 Nl40 cDNA sequences were obtained from $10 \mathrm{BPH}$ individuals. Four ORF deletions were

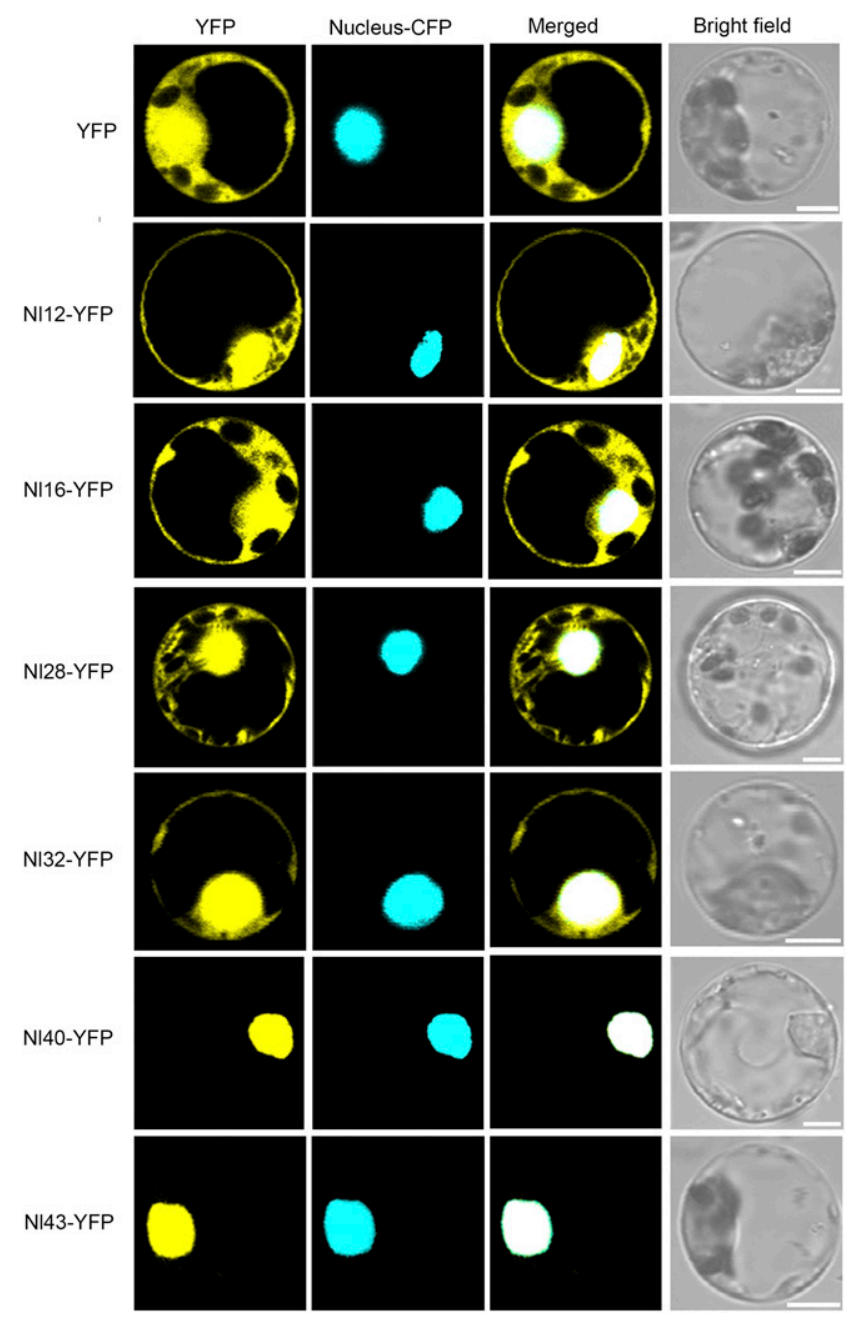

Fig. 4. Subcellular localization of brown planthopper (BPH) candidate effectors in rice protoplasts. C-terminal yellow fluorescent protein (YFP) fusion proteins were made for each of the six candidate BPH effectors lacking their endogenous signal peptides, which were then cotransformed with a cyan fluorescent protein (CFP)-tagged nuclear marker into rice protoplasts. YFP and CFP fluorescence signals were detected by confocal microscopy at $24 \mathrm{~h}$ after transformation. Scale bars $=5 \mu \mathrm{m}$. detected and various combinations of these formed seven isoforms (Supplementary Data S1). We performed a BLAST search against the BPH genome (Xue et al. 2014) with the longest Nl4O ORF version and identified that the Nl4O genomic sequence (NLH13 scaffold689, accession number $\mathrm{KN} 152627.1$ ) is composed of 12 exons and 11 introns (Fig. $6 \mathrm{~A})$. The fidelity of the $\mathrm{Nl} 40$ genomic sequence was verified by Sanger sequencing. Furthermore, we determined that the seven Nl40 isoforms arise from alternative gene splicing (Fig. 6B), which is a widespread phenomenon in eukaryotes and promotes transcriptome diversification that can potentially lead to speciation (Ast 2004). In addition to Nl40 (Nl40-1), the homolog Nl40-2 (Fig. 6C) located adjacent to Nl40 in the BPH genome has 10 isoforms (Fig. 6D) that also arise from alternative gene splicing, bringing into being a new gene family.

Nl43. N143 is an uncharacterized protein that exhibits partial similarity to only one other protein (uncharacterized protein LOC105699590, Orussus abietinus, accession number XP 012280147.1 ) in the Nr database. In addition, N143 shares $28 \%$ similarity with an alternative unigene (CL2572.Contig1_All) that also possesses a signal peptide and is unannotated in the transcriptome.

\section{BPH candidate effectors of conserved families induced} cell death with specificity.

Among the six candidate effectors, N116 and N132 are conserved in insects, N112 is conserved in eukaryotes, and two (N112 and N132) belong to conserved protein families.

$\mathrm{N} 112$ is a member of the $N$. lugens PDI (NIPDI) family. In total, 13 PDIs were identified in BPH and 5 of these presented in the $N$. lugens secretome. Thus, we examined the ability of the five NIPDI family members to induce cell death. The results showed that, whereas N112-1 induced intense cell death in Nicotiana benthamiana at 2 DPI, the remaining four NIPDI family members did not induce any symptoms (Fig. 7A), indicating that the N112-induced response is specific. Because N112-2 was not detected in $N$. benthamiana (Supplementary Fig. S7), we could not rule out the possibility that the absence of cell death is due to the instability of this protein.

The widespread CSP families in insects have been characterized to varying extents. In a previous study, 11 CSPs were identified in BPH (NlCSP1-11) (Zhou et al. 2015). To test the ability of these 11 CSPs to induce altered growth phenotype in $N$. benthamiana, we first examined the expression of these proteins in $N$. benthamiana and showed that all of them could be detected in $N$. benthamiana. Our results revealed that six CSPs (NlCSP1, NlCSP2, NlCSP4, NlCSP5, NlCSP8, and N1CSP11) induced effects in $N$. benthamiana similar to that caused by Mp10 (Fig. 7B). The remaining five CSPs did not induce a dwarf phenotype (Fig. 7B). A sequence alignment between 11 NlCSPs and Mp10 was performed using clusterx, which revealed high-level similarity among the 12 proteins (Supplementary Fig. S8).

\section{DISCUSSION}

At present, transgene or gene knockout cannot be performed in $\mathrm{BPH}$; therefore, the identification and functional analysis of effectors in BPH is not as straightforward as in plant pathogens. Nevertheless, the procedure described in this experiment proved to be effective in describing the secretome and screening for candidate effectors based on their ability to cause cell death in a nonhost plant (Fig. 8). In total, the Nilaparvata lugens salivary gland transcriptomes predicted 1,140 secreted salivary proteins that formed the BPH secretome. Out of the 64 tested salivary proteins, 6 were found to induce cell death, chlorosis, or dwarf phenotype. Four of them induced cell death 
in rice protoplasts. In other similar studies, 11 of $169 P$. sojaesecreted proteins, 7 of 95 nematode-secreted proteins, and 14 of 63 wheat pathogen-secreted proteins induced cell death or chlorosis in Nicotiana benthamiana (Chen et al. 2018; Kettles et al. 2017; Wang et al. 2011). In addition, 5 of 42 Magnaporthe oryzae-secreted proteins were shown to induce cell death in rice protoplasts (Chen et al. 2013). The number of cell-deathinducing candidate effectors in $\mathrm{BPH}$ is high, similar to that of

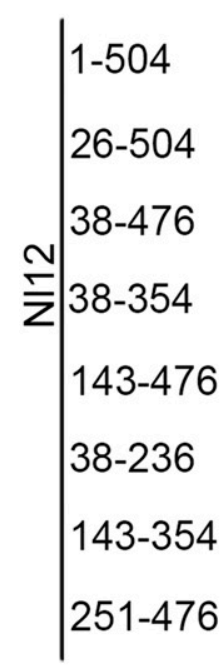

Candidate effectors and the deletion mutants

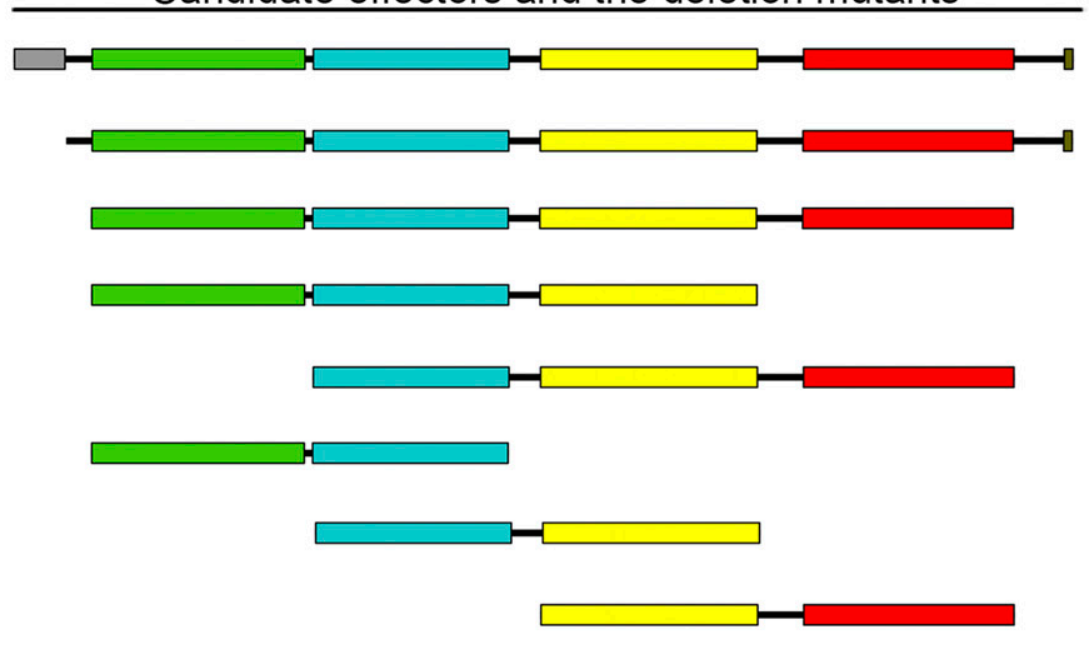

$\stackrel{\oplus}{\bar{z}} \mid \begin{aligned} & 1-189 \\ & 22-189 \\ & 30-179 \\ & 61-140 \\ & 61-130 \\ & 71-140\end{aligned}$
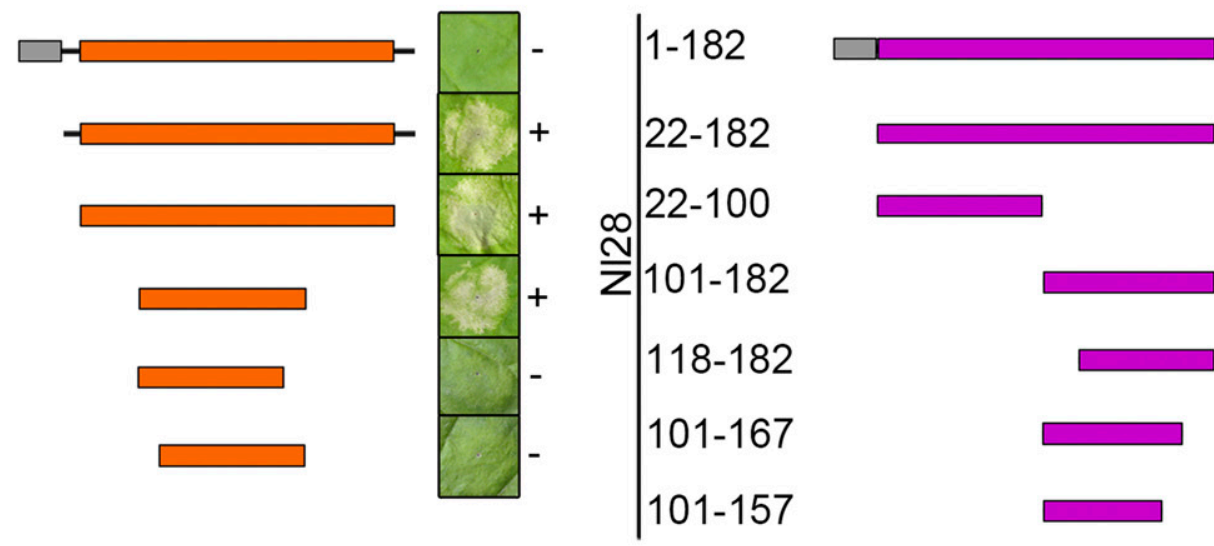

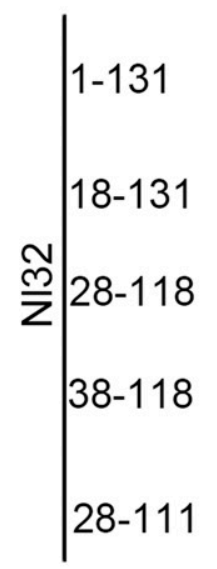
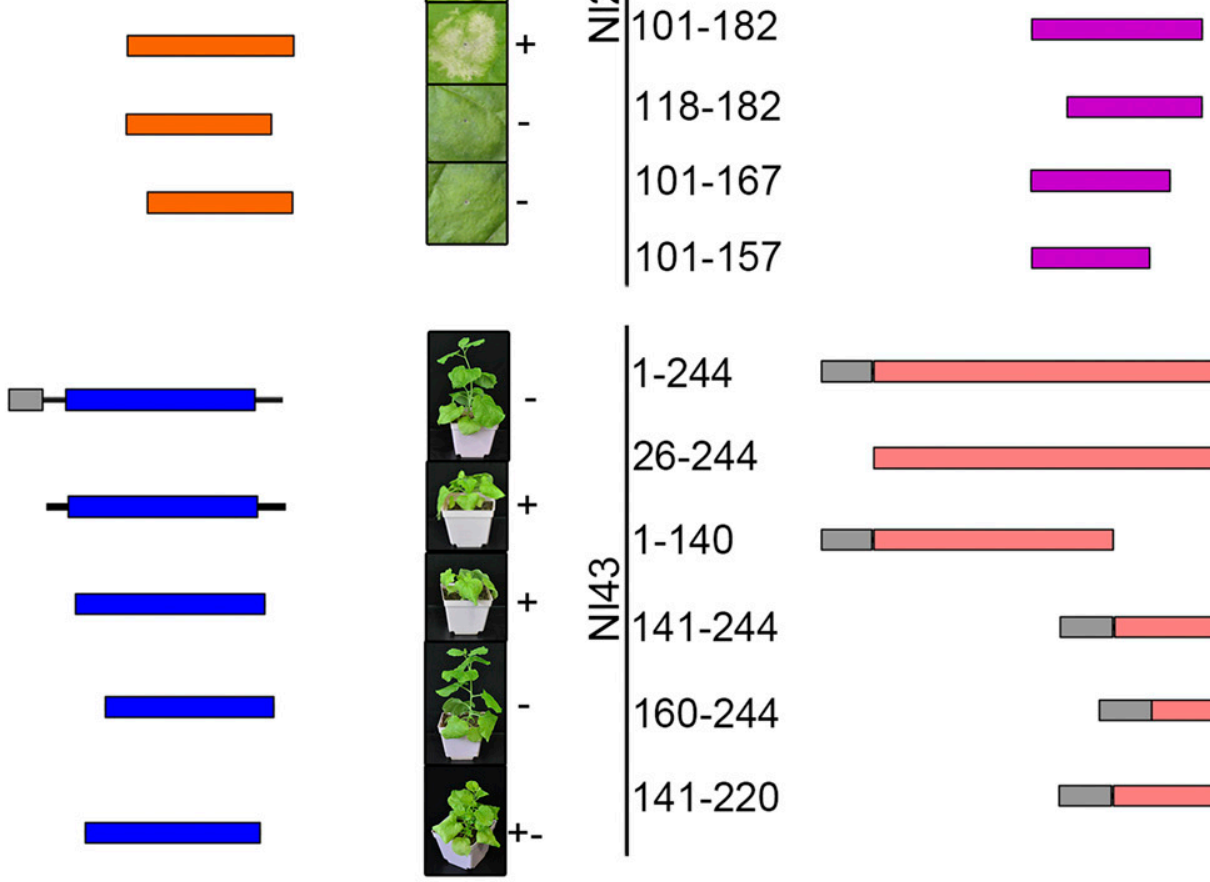

Phenotypes
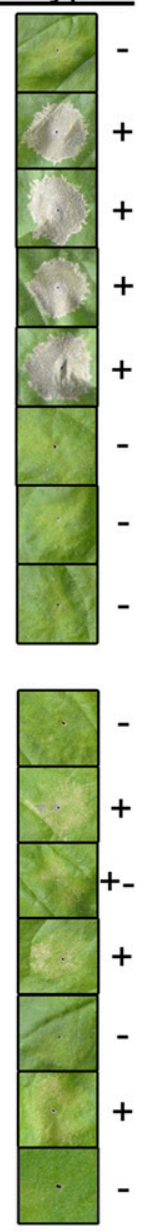

\section{Signal peptide}

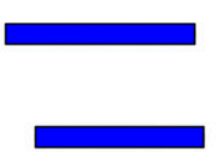

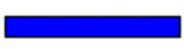

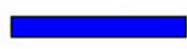

$\square$ PDI_a domain $\square$ PDI_a' domain
ER retention signal $\square$ PDI_b domain

ApoLp-III_like domain

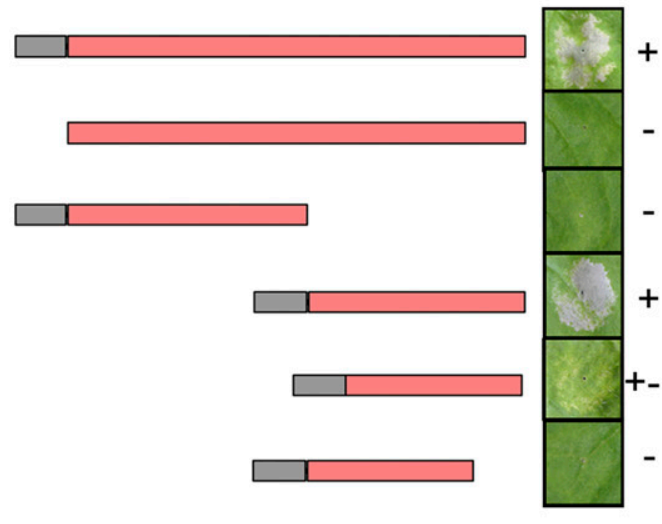

$\square$ PDI_b' domain OS-D domain

Fig. 5. Structural analysis of brown planthopper (BPH) candidate effectors that elicit plant cell death. Deletion mutants of six candidate BPH effectors were constructed and transiently expressed in Nicotiana benthamiana leaves. Amino acids numbers for residues contained in each protein version are indicated on the left side of each panel, next to their corresponding sequence structure. The corresponding plant phenotypes induced by expression of each protein version are shown on the right of each panel. The predicted domains or motifs of candidate BPH effectors are represented by different shaded boxes underneath the panels. Plant phenotypic effects were documented with a representative photo taken at 7 days postinfiltration (DPI) for N112, N116, N128, and N143, and at 15 DPI for N132. PDI = protein disulfide isomerase and ER = endoplasmic reticulum. Symbols,++- , and - indicate obvious cell death or dwarf phenotype, weak cell death or dwarf phenotype, and no cell death or dwarf phenotype, respectively. 
other plant pathogens. Based on secretome analysis and the high proportion of cell-death-inducing candidate effectors in $\mathrm{BPH}$, we propose that there is a large effector repertoire in $\mathrm{BPH}$. A large effector repertoire may simultaneously facilitate the adaptation of $\mathrm{BPH}$ to rice and production of resistance genes in rice, thus contributing to rice- $\mathrm{BPH}$ interactions and coevolution.

There are many conserved salivary proteins in the Nilaparvata lugens secretome. Of the 1,140 secreted proteins, 679 have homologs in other species. Comparative analysis revealed that 245 and 229 proteins in the $N$. lugens secretome showed homology with proteins in A. pisum and Tetranychus urticae secretome, respectively. Moreover, a number of effector proteins in other insects also display high-level conservation, such as Mp10, MIF, Armet, and ACE identified in aphids and GOX in caterpillar species. Here, among the six characterized $\mathrm{BPH}$ candidate effectors, $\mathrm{Nl12}$, N116, and $\mathrm{Nl} 32$ showed conservation among insects. The degree of effector conservation in BPH is higher than that observed for other plant pathogens. However, a number of salivary proteins may evolve quickly and are specific to $N$. lugens. Of the 1,140 secreted proteins, 461 were identified as $N$. lugens-specific proteins. Among the 100 highest expressed genes in the $N$. lugens secretome, many are salivary sheath proteins and possess numerous amino acid tandem repeats, indicating their high evolution rate and positive selection.
These highly expressed salivary proteins may play a crucial role in the rapid adaptation of BPH to rice. $N l 28, N l 40$, and $N l 43$ are $N$. lugens specific and belong to these kind of genes.

We characterized the six identified BPH candidate effectors and compared their subcellular localization, sequence, and structure features with known pathogen effectors. Of the six candidate effectors, N112, Nl40, and N143 were identified in BPH saliva by mass spectrometric analysis (Huang et al. 2016; Liu et al. 2016). All six candidate effectors showed a higher expression level in salivary glands than in other tissues. Two of the three N. lugens-specific genes, Nl4O and Nl43, were exclusively expressed in the salivary glands. In contrast, the three conserved genes N112, N116, and N132 were more extensively expressed in different tissues. The six candidate effectors localized in the nucleus or nucleus and cytoplasm of plant cell. It should be noted that small proteins may diffuse into the nucleus and the actual localization of these candidate effectors when they are delivered into the plant cell by insects might be different from when they are artificially expressed in the plant cell. Sequence analysis showed that the six candidate BPH effectors have highly diverse structures. N112, N116, N128, and N132 induced cell death without signal peptide and located in the nucleus and cytoplasm of plant cells, whereas N140 and N143 induced chlorosis or cell death with the presence of a signal peptide. Among the six BPH candidate effectors, N112, N116,

A

NI40-1

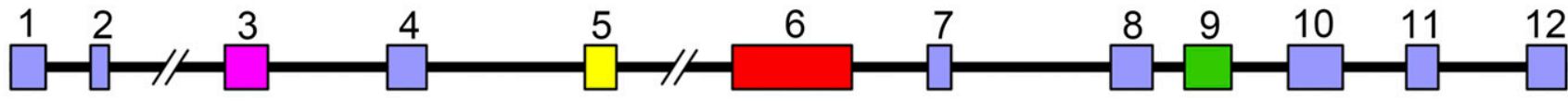

B

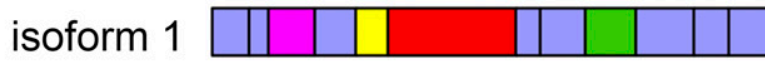

isoform 5

isoform 2

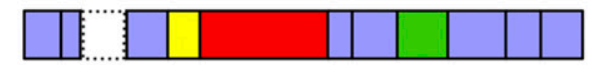

isoform 6



isoform 3

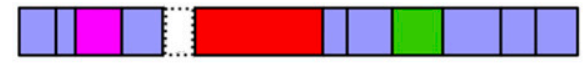

isoform 7

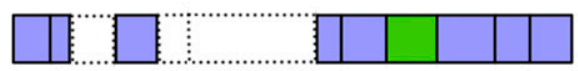

isoform 4
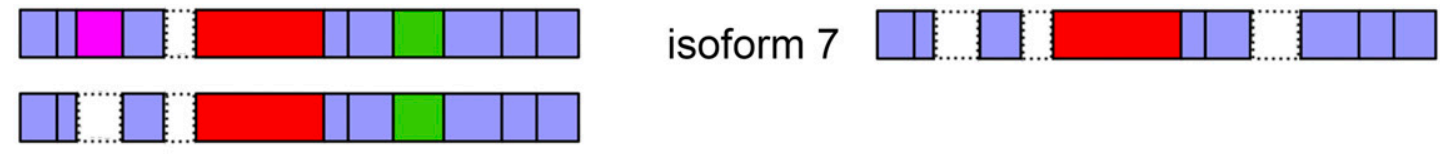

C

NI4O-2

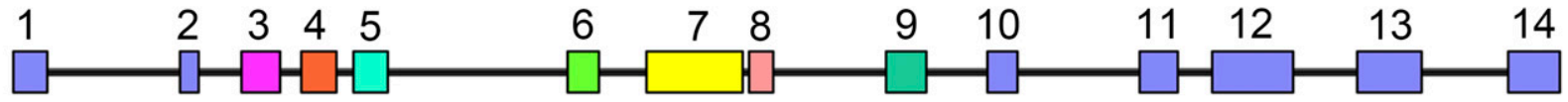

D

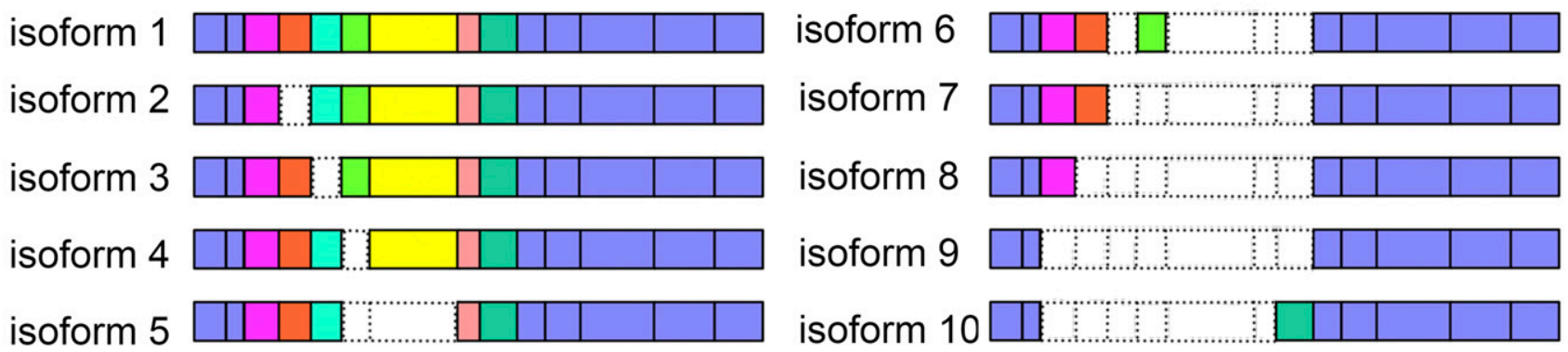

Fig. 6. Splicing patterns of genes in the N140 family. A, Genomic model of N140-1 containing 12 exons and 11 introns. Different shaded boxes represent the alternatively spliced exons $3,5,6$, and 9. B, N140-1 isoforms 1 to 7 , which represent the structure of the seven transcripts produced by alternative splicing of the four exons. White boxes indicate the positions of exons which are absent in alternatively spliced isoforms. Each exon is presented in a size in proportion to its sequence length. C, Genomic model of N140-2 containing 14 exons and 13 introns. Different shaded boxes represent the seven alternatively spliced exons 3 to 9. D, N140-2 isoforms 1 to 10, which represent the structure of the 10 transcripts produced by alternative splicing of the seven exons. White boxes indicate the positions of exons which are absent in alternatively spliced isoforms. Each exon is presented in a size in proportion to its sequence length. 
and N132 display protein conservation and two (N112 and N132) belong to large gene families. We did not identify an N128 homolog in the NCBI database, and found N128 to resemble SSCPs which are commonly fungal effectors. N143 exhibits partial similarity to only one other protein in the $\mathrm{Nr}$ database. We did not identify homologs of the Nl4O family in other organisms; however, there are two Nl40 family members in the $\mathrm{BPH}$ genome and a variety of isoforms in the transcriptome arising from alternative splicing. As a multicellular eukaryote, alternative splicing is more prevalent in BPH compared with bacteria, fungi, and oomycetes (Ast 2004), thus increasing the diversity within the effector repertoire and contributing to the complex interaction between $\mathrm{BPH}$ and rice.

N112 is a member of the PDI. In recent years, PDIs have been identified in the saliva of a number of insects such as ticks and BPH (Huang et al. 2016; Liao et al. 2007; Tirloni et al. 2015). Pathogen PDIs are important virulence factors that function during infection (Stolf et al. 2011). PpPDI1, the $P$. parasitica N112 effector homolog, induces substantial cell death in $\mathrm{Ni}$ cotiana benthamiana. In addition, overexpression of PpPDI enhances $P$. parasitica virulence during infection (Meng et al. 2015). N116 is an apolipophorin-III protein. Apolipophorin-III belongs to a large family of exchangeable apolipoproteins that play vital roles in lipid transport and lipoprotein metabolism (Wang et al. 2002). Recently, apolipophorin was identified in the saliva of rice leafhopper and aphids and in the salivary glands of planthoppers (Konishi et al. 2009; Nicholson et al. 2012; Vandermoten et al. 2014). Lipids and free fatty acids, which function as plant defense signaling compounds, may be potential targets for apolipophorin (Madey et al. 2002). As for N132, recent findings have revealed novel functions of CSPs. In two Helicoverpa spp., CSP4 is uniquely and abundantly expressed in the proboscis and, once secreted, acts as a surfactant to reduce the surface tension of aqueous solutions, thus reducing the pressure of feeding (Liu et al. 2014). In M. persicae, the chemosensory protein Mp10 was shown to be a salivary effector protein that was delivered into the plant mesophyll cell cytosol during feeding, where it triggered plant defense responses, thus reducing aphid fecundity (Bos et al. 2010; Mugford et al. 2016; Rodriguez et al. 2014). Our findings suggest that N112 and N132 may share similar functions with their homologous effectors in $N$. benthamiana and that distinct sap-feeding insects may share a number of salivary proteins and common mechanisms to disarm plant defense systems.
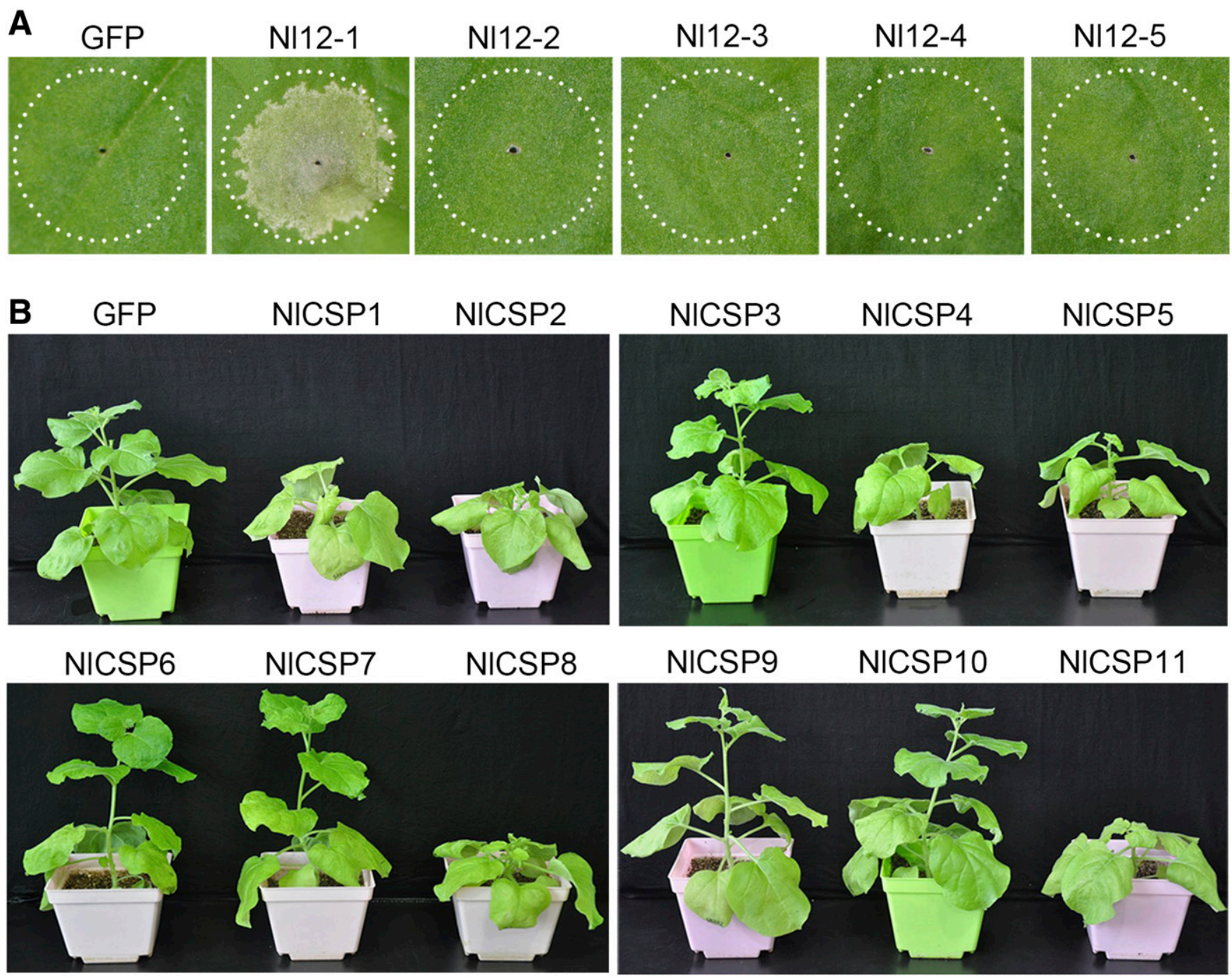

Fig. 7. Members of two brown planthopper (BPH) candidate effector families induce cell death in a specific manner in Nicotiana benthamiana. A, Five members of the NIPDI family were transiently expressed in $N$. benthamiana leaves via agroinfiltration. A single member (N112-1) of the NlPDI family induced cell death. B, In all, 11 members of NlCSP family were transiently expressed in N. benthamiana leaves. Six members (NICSP1, NlCSP2, NlCSP4, NlCSP5, NICSP8, and NICSP11) of the NlCSP family induced a dwarf phenotype. Green fluorescent protein (GFP) was infiltrated as a control. The effect of each BPH candidate effector on plant phenotype was documented with a representative photo taken at 7 days postinfiltration (DPI) for the NIPDI family and at 15 DPI for the NICSP family. 
We observed that BPH salivary proteins N112, N116, N128, and N143 induced cell death in rice protoplasts and N. benthamiana leaves, and immunity responses in N. benthamiana, reflecting some sort of conserved recognition by plant immune receptors. However, the exact function of these BPH candidate effectors and recognition of the mechanism in plants remain to be elucidated. Although these BPH candidate effectors may induce plant defense responses, these responses may be suppressed by other effectors, thus preventing any adverse impact on the BPH survival. Moreover, it should be noted that nonhost plants may react to effectors more frequently and intensely than host plants (Wroblewski et al. 2009) and, at present, there is no evidence that cell death occurs in the process of rice defense against $\mathrm{BPH}$. The Nilaparvata lugens secretome described here provides a valuable platform to identify effectors that suppress rice defense systems and those that interact with rice resistance genes.

\section{MATERIALS AND METHODS}

\section{Sequence analysis.}

PDIs from the following species were obtained from GenBank: P. parasitica (XP_008914616.1), M. oryzae (XP 003710672.1), Oryza sativa (XP_015627045.1), Nicotiana benthamiana (XP_019255435.1), A. pisum (XP_008183165.1), and Nilaparvata lugens (ANJ04677.1). Apolipophorin-III sequences from the following seven insect species were downloaded from the Swiss-Prot database: Pararge aegeria (S4PD79), Epiphyas postvittana (Q9GPN5), Danaus plexippus
(G6CIC8), Apis cerana (V9ILX0), Dufourea novaeangliae (A0A154PL18), and Triatoma infestans (A0A170YUN3). The transcriptomes of six insect species (Acyrthosiphon pisum, Tribolium castaneum, Drosophila melanogaster, Nasonia vitripennis, Manduca sexta, and L. migratoria) were obtained from Ensembl Genomes databases. Amino acid sequence alignment of the six PDIs and the 12 CSPs was performed using ClustalX 2.1 (Larkin et al. 2007).

\section{Salivary gland collection and Illumina sequencing.}

Salivary glands were dissected from BPH adult females on ice following a previously described procedure (Shangguan et al. 2018). Approximately 100 salivary glands for each of the five BPH populations were collected. Total RNA of salivary glands was isolated using the RNAiso Plus kit (TaKaRa) according to the manufacturer's protocol. The RNA samples were shipped on dry ice to Beijing Genomics Institute, and subsequently sequenced on the Illumina Hiseq 2000 platform.

Prediction of the BPH secretome and protein clustering.

The unigenes were aligned using BLASTX (E-value < $0.00001)$ to the protein databases Nr, Swiss-Prot, and KEGG. The BLAST results were used to extract coding sequences (CDSs) of unigenes. The CDSs of unigenes with no BLAST hits were predicted by ESTScan (Iseli et al. 1999). Signal peptide prediction was carried out using SignalP 4.1 (Petersen et al. 2011). Subsequently, transmembrane domains were predicted using THMMM v2.0 (Krogh et al. 2001). Proteins

A

Secretome prediction

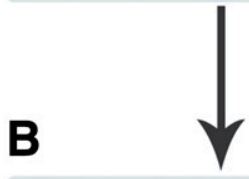

Secretome analysis

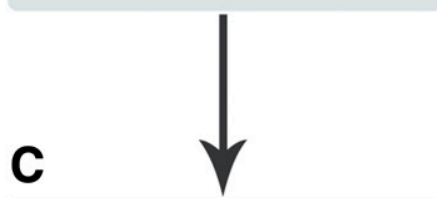

Candidate effector selection criteria

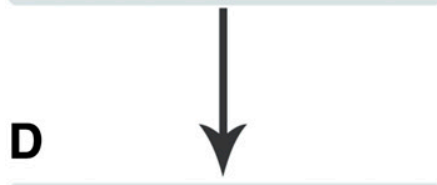

Effector verification (a) Salivary gland transcriptome sequencing and assembly

(b) Signal peptide prediction

(c) Transmembrane domain prediction

(a) Gene expression analysis

(b) Clustering protein families

(c) Identification of conserved and $N$. lugens-specific salivary proteins

(d) Identification of amino acid tandem repeats and cysteine-rich proteins

(a) Highly expressed in salivary glands

(b) Member of a large protein family

(c) Homolog of known effectors

(d) Rapidly evolving genes or N. lugens-specific genes

Transiently expressing candidate effectors in planta to determine whether they induce cell death or other phenotypes, elicit plant defense responses, such as expression of defense-related genes and callose deposition

Fig. 8. Pipeline for the identification of brown planthopper (BPH) candidate effectors. A, Bioinformatics pipeline for the prediction of secreted proteins following salivary gland transcriptome sequencings. B, Secretome analysis for the identification of candidate effectors. C, Selection criteria applied to candidate effectors to identify most-likely functional effectors. D, Experimental methodology to verify the functionality of effectors. 
that had a signal peptide and a 0 -to- 1 transmembrane domain (the transmembrane domain being in the signal peptide) were considered as secreted proteins. The secretome was then submitted to generate protein families using OrthoMCL v2.0 ( $\mathrm{Li}$ et al. 2003) with standard parameters.

\section{Cloning of candidate BPH effectors and plasmid construction.}

To clone the candidate BPH effectors, primers were designed for the corresponding ORFs. Sequences were amplified from cDNA prepared from 10 whole insect bodies of BPH biotype I, then ligated into a pMD18-T vector. For construction of the PVX vectors, two fragment versions (ORF+SP and ORF-SP) were amplified and ligated into a pGR107 vector. For construction of the Gateway entry clones, primers were designed to amplify genes without a stop codon. PCR products were inserted into pDONR207 via BP reactions (Gateway BP clonase enzyme mix; Invitrogen), then cloned into the plant expression vectors pEarleyGate100 and pEarleyGate101 which contain the CaMV 35S promoter via LR reactions (Gateway LR clonase enzyme mix; Invitrogen). The resulting pEarleyGate101 constructs were used for subcellular localization of candidate effectors in rice protoplasts and $N$. benthamiana. The resulting pEarleyGate100 constructs was used for callose deposition assays, cell death assays of candidate effectors, and analysis of deletion mutants and effector families in Nicotiana benthamiana. For Nl32 and structural analysis of $N l 28$, we used the PVX vector. Deletion mutants of NI43 were produced by overlapping PCR. Details of constructs and primers (including primers used for expression analysis of defense-related genes) are listed in Supplementary Table S8.

\section{Transient expression in $N$. benthamiana.}

Constructs were introduced into Agrobacterium tumefaciens GV3101 by electroporation. Transformed GV3101 strains were grown at $28^{\circ} \mathrm{C}$ for $24 \mathrm{~h}$. The cultured Agrobacterium cells were harvested by centrifugation, resuspended in infiltration buffer (10 $\mathrm{mM} \mathrm{MgCl} 2,10 \mathrm{mM}$ morpholineethanesulfonic acid, and $150 \mathrm{mM}$ acetosyringone) to an optical density at $600 \mathrm{~nm}$ of 0.6 , and incubated at $28^{\circ} \mathrm{C}$ for 1 to $3 \mathrm{~h}$ before infiltration. $A$. tumefaciens cell suspensions were infiltrated using a needleless syringe into the leaves of $N$. benthamiana plants grown in a greenhouse for 4 to 6 weeks.

\section{Protein extraction and immunoblot analysis.}

At $48 \mathrm{~h}$ following agroinfiltration in $N$. benthamiana leaves, total protein extracts were prepared by homogenizing $400 \mathrm{mg}$ of leaf tissue in $1 \mathrm{ml}$ of radio immunoprecipitation assay buffer (Beyotime) in the presence of $0.1 \mathrm{mM}$ protease inhibitor phenylmethylsulfonyl fluoride (Beyotime). Proteins samples were separated by sodium dodecyl sulfate polyacrylamide gel electrophoresis and then transferred onto nitrocellulose membranes. Immunoblot analysis was performed as previously described $(\mathrm{Hu}$ et al. 2011). Protein expression was detected using anti-HA tag antibodies.

\section{Callose deposition assays in $N$. benthamiana.}

Callose deposition assays in $N$. benthamiana were performed as previously described (Naessens et al. 2015), with some modifications. Leaf discs were mounted in $80 \%$ glycerol and observed under a laser-scanning confocal microscope (Olympus Fluoview FV1000). Images were analyzed using the ImageJ software.

\section{RNA isolation and RT-qPCR.}

Different tissues, including salivary glands, midguts, fat bodies, and the remaining parts, were dissected from $\mathrm{BPH}$ adults under a stereomicroscope. For BPH RNA extraction, total RNA of whole insect bodies and different tissues was isolated using an RNAiso Plus kit (TaKaRa) according to the manufacturer's instructions. Total RNA was isolated from $N$. benthamiana leaves expressing various constructs using an EASYspin tissue/cell RNA rapid extraction kit (Yuanpinghao Biotech). cDNA was synthesized using a PrimeScript RT reagent kit (TaKaRa). For RT-qPCR assays, GFP was infiltrated as a control. Expression levels of target genes were normalized to the housekeeping gene $\beta$-actin. Relative expression ratios were calculated using the Pfaffl method (Pfaffl 2001). RTqPCR was performed using a Bio-Rad CFX96 Real-Time System (Applied Biosystems).

\section{Cell death assays and subcellular localization in rice protoplasts.}

Transient expression in rice protoplasts was carried out as previously described (Chen et al. 2006). The firefly luciferase gene (LUC) was used as a reporter that reflected protoplast viability. Constructs of candidate effectors under the control of the maize ubiquitin promoter were cotransformed alongside the LUC gene into rice protoplasts by polyethylene-glycol-mediated transformation (Chen et al. 2006). Luciferase activity was detected $40 \mathrm{~h}$ following transformation using a luciferase assay system (Promega Corp.), and the reduction in LUC signal was compared with that in GFP-transformed protoplasts.

For subcellular localization, the pCXUN-effector-YFP constructs were cotransformed with the cyan fluorescent proteinnuclear marker bZIP63 (Walter et al. 2004) in rice protoplasts. At 16 to $24 \mathrm{~h}$ following transformation, protoplasts were observed using a laser-scanning confocal microscope (Olympus Fluoview FV1000).

\section{ACKNOWLEDGMENTS}

We thank Y. Wang at Nanjing Agricultural University for providing the pGR107 vector and G. Genedenovo Biotechnology for gene annotation.

\section{LITERATURE CITED}

Ast, G. 2004. How did alternative splicing evolve? Nat. Rev. Genet. 5: $773-782$.

Augustijn, K. D., Kleemann, R., Thompson, J., Kooistra, T., Crawford, C. E., Reece, S. E., Pain, A., Siebum, A. H. G., Janse, C. J., and Waters, A. P. 2007. Functional characterization of the Plasmodium falciparum and $P$. berghei homologues of macrophage migration inhibitory factor Infect. Immun. 75:1116-1128.

Ben Achour, Y., Chenik, M., Louzir, H., and Dellagi, K. 2002. Identification of a disulfide isomerase protein of Leishmania major as a putative virulence factor. Infect. Immun. 70:3576-3585.

Bos, J. I., Prince, D., Pitino, M., Maffei, M. E., Win, J., and Hogenhout, S. A. 2010. A functional genomics approach identifies candidate effectors from the aphid species Myzus persicae (green peach aphid). PLoS Genet. 6:e1001216.

Bowen, C. J., Jaworski, D. C., Wasala, N. B., and Coons, L. B. 2010. Macrophage migration inhibitory factor expression and protein localization in Amblyomma americanum (Ixodidae). Exp. Appl. Acarol. 50:343-352.

Caillaud, M. C., Piquerez, S. J. M., Fabro, G., Steinbrenner, J., Ishaque, N., Beynon, J., and Jones, J. D. G. 2012. Subcellular localization of the Hpa RxLR effector repertoire identifies a tonoplast-associated protein HaRxL17 that confers enhanced plant susceptibility. Plant J. 69: 252-265.

Carolan, J. C., Caragea, D., Reardon, K. T., Mutti, N. S., Dittmer, N., Pappan, K., Cui, F., Castaneto, M., Poulain, J., Dossat, C., Tagu, D., Reese, J. C., Reeck, G. R., Wilkinson, T. L., and Edwards, O. R. 2011 Predicted effector molecules in the salivary secretome of the pea aphid (Acyrthosiphon pisum): A dual transcriptomic/proteomic approach. J. Proteome Res. 10:1505-1518.

Chen, C., Chen, Y., Jian, H., Yang, D., Dai, Y., Pan, L., Shi, F., Yang, S., and Liu, Q. 2018. Large-scale identification and characterization of Heterodera avenae putative effectors suppressing or inducing cell death in Nicotiana benthamiana. Front. Plant Sci. 8:2062. 
Chen, S., Songkumarn, P., Venu, R. C., Gowda, M., Bellizzi, M., Hu, J., Liu, W., Ebbole, D., Meyers, B., Mitchell, T., and Wang, G. L. 2013. Identification and characterization of in planta-expressed secreted effector proteins from Magnaporthe oryzae that induce cell death in rice. Mol. Plant-Microbe Interact. 26:191-202.

Chen, S., Tao, L., Zeng, L., Vega-Sanchez, M. E., Umemura, K., and Wang, G. L. 2006. A highly efficient transient protoplast system for analyzing defence gene expression and protein-protein interactions in rice. Mol. Plant Pathol. 7:417-427.

Cherqui, A., and Tjallingii, W. F. 2000. Salivary proteins of aphids, a pilot study on identification, separation and immunolocalisation. J. Insect Physiol. 46:1177-1186.

Dietrich, R. A., Delaney, T. P., Uknes, S. J., Ward, E. R., Ryals, J. A., and Dangl, J. L. 1994. Arabidopsis mutants simulating disease resistance response. Cell 77:565-577.

Dowen, R. H., Engel, J. L., Shao, F., Ecker, J. R., and Dixon, J. E. 2009. A family of bacterial cysteine protease type III effectors utilizes acylationdependent and -independent strategies to localize to plasma membranes. J. Biol. Chem. 284:15867-15879.

Eichenseer, H., Mathews, M. C., Powell, J. S., and Felton, G. W. 2010. Survey of a salivary effector in caterpillars: Glucose oxidase variation and correlation with host range. J. Chem. Ecol. 36:885-897.

Guiguet, A., Dubreuil, G., Harris, M. O., Appel, H. M., Schultz, J. C., Pereira, M. H., and Giron, D. 2016. Shared weapons of blood- and plantfeeding insects: Surprising commonalities for manipulating hosts. J. Insect Physiol. 84:4-21.

Guyon, K., Balagué, C., Roby, D., and Raffaele, S. 2014. Secretome analysis reveals effector candidates associated with broad host range necrotrophy in the fungal plant pathogen Sclerotinia sclerotiorum. BMC Genomics 15:336.

Haas, B. J., Kamoun, S., Zody, M. C., Jiang, R. H. Y., Handsaker, R. E., Cano, L. M., Grabherr, M., Kodira, C. D., Raffaele, S., Torto-Alalibo, T., Bozkurt, T. O., Ah-Fong, A. M. V., Alvarado, L., Anderson, V. L., Armstrong, M. R., Avrova, A., Baxter, L., Beynon, J., Boevink, P. C., Bollmann, S. R., Bos, J. I. B., Bulone, V., Cai, G., Cakir, C., Carrington, J. C., Chawner, M., Conti, L., Costanzo, S., Ewan, R., Fahlgren, N., Fischbach, M. A., Fugelstad, J., Gilroy, E. M., Gnerre, S., Green, P. J., Grenville-Briggs, L. J., Griffith, J., Grünwald, N. J., Horn, K., Horner, N. R., Hu, C. H., Huitema, E., Jeong, D. H., Jones, A. M. E., Jones, J. D. G., Jones, R. W., Karlsson, E. K., Kunjeti, S. G., Lamour, K., Liu, Z., Ma, L., Maclean, D., Chibucos, M. C., McDonald, H., McWalters, J., Meijer, H. J. G., Morgan, W., Morris, P. F., Munro, C. A., O’Neill, K., Ospina-Giraldo, M., Pinzón, A., Pritchard, L., Ramsahoye, B., Ren, Q., Restrepo, S., Roy, S., Sadanandom, A., Savidor, A., Schornack, S., Schwartz, D. C., Schumann, U. D., Schwessinger, B., Seyer, L., Sharpe, T., Silvar, C., Song, J., Studholme, D. J., Sykes, S., Thines, M., van de Vondervoort, P. J., Phuntumart, V., Wawra, S., Weide, R., Win, J., Young, C., Zhou, S., Fry, W., Meyers, B. C., van West, P., Ristaino, J., Govers, F., Birch, P. R., Whisson, S. C., Judelson, H. S., and Nusbaum, C. 2009. Genome sequence and analysis of the Irish potato famine pathogen Phytophthora infestans. Nature 461:393-398.

Harmel, N., Létocart, E., Cherqui, A., Giordanengo, P., Mazzucchelli, G., Guillonneau, F., De Pauw, E., Haubruge, E., and Francis, F. 2008. Identification of aphid salivary proteins: A proteomic investigation of Myzus persicae. Insect Mol. Biol. 17:165-174.

Hogenhout, S. A., Van der Hoorn, R. A., Terauchi, R., and Kamoun, S. 2009. Emerging concepts in effector biology of plant-associated organisms. Mol. Plant-Microbe Interact. 22:115-122.

Hu, J., Zhou, J., Peng, X., Xu, H., Liu, C., Du, B., Yuan, H., Zhu, L., and He, G. 2011. The Bphi008a gene interacts with the ethylene pathway and transcriptionally regulates $M A P K$ genes in the response of rice to brown planthopper feeding. Plant Physiol. 156:856-872.

Huang, H. J., Liu, C. W., Huang, X. H., Zhou, X., Zhuo, J. C., Zhang, C. X., and Bao, Y. Y. 2016. Screening and functional analyses of Nilaparvata lugens salivary proteome. J. Proteome Res. 15:1883-1896.

Huang, H. J., Liu, C. W., Xu, H. J., Bao, Y. Y., and Zhang, C. X. 2017. Mucin-like protein, a saliva component involved in brown planthopper virulence and host adaptation. J. Insect Physiol. 98:223-230.

Huntley, M., and Golding, G. B. 2000. Evolution of simple sequence in proteins. J. Mol. Evol. 51:131-140.

Iseli, C., Jongeneel, C. V., and Bucher, P. 1999. ESTScan: A program for detecting, evaluating, and reconstructing potential coding regions in EST sequences. Pages 138-148 in: Proc. Int. Conf. Intell. Syst. Mol. Biol.

Jaouannet, M., Rodriguez, P. A., Thorpe, P., Lenoir, C. J. G., MacLeod, R., Escudero-Martinez, C., and Bos, J. I. B. 2014. Plant immunity in plantaphid interactions. Front. Plant Sci. 5:663.
Ji, R., Ye, W., Chen, H., Zeng, J., Li, H., Yu, H., Li, J., and Lou, Y. 2017. A salivary endo- $\beta$-1,4-glucanase acts as an effector that enables the brown planthopper to feed on rice. Plant Physiol. 173:1920-1932.

Ji, R., Yu, H., Fu, Q., Chen, H., Ye, W., Li, S., and Lou, Y. 2013. Comparative transcriptome analysis of salivary glands of two populations of rice brown planthopper, Nilaparvata lugens, that differ in virulence. PLoS One 8:e79612.

Kaloshian, I., and Walling, L. L. 2016. Hemipteran and dipteran pests: Effectors and plant host immune regulators. J. Integr. Plant Biol. 58: 350-361.

Keller, H., Blein, J. P., Bonnet, P., and Ricci, P. 1996. Physiological and molecular characteristics of elicitin-induced systemic acquired resistance in tobacco. Plant Physiol. 110:365-376.

Kettles, G. J., Bayon, C., Canning, G., Rudd, J. J., and Kanyuka, K. 2017. Apoplastic recognition of multiple candidate effectors from the wheat pathogen Zymoseptoria tritici in the nonhost plant Nicotiana benthamiana. New Phytol. 213:338-350.

Konishi, H., Noda, H., Tamura, Y., and Hattori, M. 2009. Proteomic analysis of the salivary glands of the rice brown planthopper, Nilaparvata lugens (Stål) (Homoptera: Delphacidae). Appl. Entomol. Zool. (Jpn.) 44:525-534.

Krogh, A., Larsson, B., von Heijne, G., and Sonnhammer, E. L. 2001 Predicting transmembrane protein topology with a hidden Markov model: Application to complete genomes. J. Mol. Biol. 305:567-580.

Kuśnierczyk, A., Winge, P., Jørstad, T. S., Troczyńska, J., Rossiter, J. T., and Bones, A. M. 2008. Towards global understanding of plant defence against aphids-Timing and dynamics of early Arabidopsis defence responses to cabbage aphid (Brevicoryne brassicae) attack. Plant Cell Environ. 31:1097-1115.

Larkin, M. A., Blackshields, G., Brown, N. P., Chenna, R., McGettigan, P. A., McWilliam, H., Valentin, F., Wallace, I. M., Wilm, A., Lopez, R., Thompson, J. D., Gibson, T. J., and Higgins, D. G. 2007. Clustal W and Clustal X version 2.0. Bioinformatics 23:2947-2948.

Li, L., Stoeckert, C. J., Jr., and Roos, D. S. 2003. OrthoMCL: Identification of ortholog groups for eukaryotic genomes. Genome Res. 13:2178-2189.

Li, R., Yu, C., Li, Y., Lam, T. W., Yiu, S. M., Kristiansen, K., and Wang, J. 2009. SOAP2: An improved ultrafast tool for short read alignment. Bioinformatics 25:1966-1967.

Liao, M., Hatta, T., Umemiya, R., Huang, P., Jia, H., Gong, H., Zhou, J., Nishikawa, Y., Xuan, X., and Fujisaki, K. 2007. Identification of three protein disulfide isomerase members from Haemaphysalis longicornis tick. Insect Biochem. Mol. Biol. 37:641-654.

Liu, X., Zhou, H., Zhao, J., Hua, H., and He, Y. 2016. Identification of the secreted watery saliva proteins of the rice brown planthopper, Nilaparvata lugens (Stål) by transcriptome and Shotgun LC-MS/MS approach. J. Insect Physiol. 89:60-69.

Liu, Y. L., Guo, H., Huang, L. Q., Pelosi, P., and Wang, C. Z. 2014. Unique function of a chemosensory protein in the proboscis of two Helicoverpa species. J. Exp. Biol. 217:1821-1826.

Luna, E., Pastor, V., Robert, J., Flors, V., Mauch-Mani, B., and Ton, J. 2011. Callose deposition: A multifaceted plant defense response. Mol. PlantMicrobe Interact. 24:183-193.

Madey, E., Nowack, L. M., and Thompson, J. E. 2002. Isolation and characterization of lipid in phloem sap of canola. Planta 214:625-634.

Maimbo, M., Ohnishi, K., Hikichi, Y., Yoshioka, H., and Kiba, A. 2010. Sglycoprotein-like protein regulates defense responses in Nicotiana plants against Ralstonia solanacearum. Plant Physiol. 152:2023-2035.

Meng, Y., Zhang, Q., Zhang, M., Gu, B., Huang, G., Wang, Q., and Shan, W. 2015. The protein disulfide isomerase 1 of Phytophthora parasitica (PpPDI1) is associated with the haustoria-like structures and contributes to plant infection. Front. Plant Sci. 6:632.

Miles, P. W. 1999. Aphid saliva. Biol. Rev. 74:41-85. link

Mugford, S. T., Barclay, E., Drurey, C., Findlay, K. C., and Hogenhout, S. A. 2016. An immuno-suppressive aphid saliva protein is delivered into the cytosol of plant mesophyll cells during feeding. Mol. Plant-Microbe Interact. 29:854-861.

Mularoni, L., Ledda, A., Toll-Riera, M., and Albà, M. M. 2010. Natural selection drives the accumulation of amino acid tandem repeats in human proteins. Genome Res. 20:745-754.

Musser, R. O., Hum-Musser, S. M., Eichenseer, H., Peiffer, M., Ervin, G., Murphy, J. B., and Felton, G. W. 2002. Caterpillar saliva beats plant defences. Nature 416:599-600.

Mutti, N. S., Louis, J., Pappan, L. K., Pappan, K., Begum, K., Chen, M. S., Park, Y., Dittmer, N., Marshall, J., Reese, J. C., and Reeck, G. R. 2008. A protein from the salivary glands of the pea aphid, Acyrthosiphon pisum, is essential in feeding on a host plant. Proc. Natl. Acad. Sci. U.S.A. 105: 9965-9969 
Naessens, E., Dubreuil, G., Giordanengo, P., Baron, O. L., Minet-Kebdani, N., Keller, H., and Coustau, C. 2015. A secreted MIF cytokine enables aphid feeding and represses plant immune responses. Curr. Biol. 25: 1898-1903.

Nicholson, S. J., Hartson, S. D., and Puterka, G. J. 2012. Proteomic analysis of secreted saliva from Russian wheat aphid (Diuraphis noxia Kurd.) biotypes that differ in virulence to wheat. J. Proteomics 75:2252-2268.

Pelosi, P., Calvello, M., and Ban, L. 2005. Diversity of odorant-binding proteins and chemosensory proteins in insects. Chem. Senses 30: i291-i292.

Petersen, T. N., Brunak, S., von Heijne, G., and Nielsen, H. 2011. SignalP 4.0: Discriminating signal peptides from transmembrane regions. Nat. Methods 8:785-786.

Pfaffl, M. W. 2001. A new mathematical model for relative quantification in real-time RT-PCR. Nucleic Acids Res. 29:e45.

Raffaele, S., Farrer, R. A., Cano, L. M., Studholme, D. J., MacLean, D., Thines, M., Jiang, R. H., Zody, M. C., Kunjeti, S. G., Donofrio, N. M. Meyers, B. C., Nusbaum, C., and Kamoun, S. 2010. Genome evolution following host jumps in the Irish potato famine pathogen lineage. Science 330:1540-1543.

Rodriguez, P. A., Stam, R., Warbroek, T., and Bos, J. I. B. 2014. Mp10 and Mp42 from the aphid species Myzus persicae trigger plant defenses in Nicotiana benthamiana through different activities. Mol. PlantMicrobe Interact. 27:30-39.

Saunders, D. G., Win, J., Cano, L. M., Szabo, L. J., Kamoun, S., and Raffaele, S. 2012. Using hierarchical clustering of secreted protein families to classify and rank candidate effectors of rust fungi. PLoS One 7:e29847.

Schornack, S., van Damme, M., Bozkurt, T. O., Cano, L. M., Smoker, M., Thines, M., Gaulin, E., Kamoun, S., and Huitema, E. 2010. Ancient class of translocated oomycete effectors targets the host nucleus. Proc. Natl. Acad. Sci. U.S.A. 107:17421-17426.

Sezer, M., and Butlin, R. K. 1998. The genetic basis of host plant adaptation in the brown planthopper (Nilaparvata lugens). Heredity 80:499-508.

Shangguan, X., Zhang, J., Liu, B., Zhao, Y., Wang, H., Wang, Z., Guo, J., Rao, W., Jing, S., Guan, W., Ma, Y., Wu, Y., Hu, L., Chen, R., Du, B., Zhu, L., Yu, D., and He, G. 2018. A mucin-like protein of planthopper is required for feeding and induces immunity response in plants. Plant Physiol. 176:552-565.

Stergiopoulos, I., and de Wit, P. J. 2009. Fungal effector proteins. Annu. Rev. Phytopathol. 47:233-263.

Stolf, B. S., Smyrnias, I., Lopes, L. R., Vendramin, A., Goto, H., Laurindo, F. R. M., Shah, A. M., and Santos, C. X. C. 2011. Protein disulfide isomerase and host-pathogen interaction. Sci. World J. 11:1749-1761.

Tirloni, L., Islam, M. S., Kim, T. K., Diedrich, J. K., Yates, J. R., 3rd, Pinto, A. F. M., Mulenga, A., You, M. J., and Da Silva Vaz, I., Jr. 2015. Saliva from nymph and adult females of Haemaphysalis longicornis: A proteomic study. Parasit. Vectors 8:338.

Vandermoten, S., Harmel, N., Mazzucchelli, G., De Pauw, E., Haubruge, E., and Francis, F. 2014. Comparative analyses of salivary proteins from three aphid species. Insect Mol. Biol. 23:67-77.

Verma, S., Gazara, R. K., Nizam, S., Parween, S., Chattopadhyay, D., and Verma, P. K. 2016. Draft genome sequencing and secretome analysis of fungal phytopathogen Ascochyta rabiei provides insight into the necrotrophic effector repertoire. Sci. Rep. 6: Article 24638.

Vermeire, J. J., Cho, Y., Lolis, E., Bucala, R., and Cappello, M. 2008. Orthologs of macrophage migration inhibitory factor from parasitic nematodes. Trends Parasitol. 24:355-363.
Villarroel, C. A., Jonckheere, W., Alba, J. M., Glas, J. J., Dermauw, W., Haring, M. A., Van Leeuwen, T., Schuurink, R. C., and Kant, M. R. 2016. Salivary proteins of spider mites suppress defenses in Nicotiana benthamiana and promote mite reproduction. Plant J. 86:119-131.

Voigt, C. A. 2014. Callose-mediated resistance to pathogenic intruders in plant defense-related papillae. Front. Plant Sci. 5:168.

Walter, M., Chaban, C., Schütze, K., Batistic, O., Weckermann, K., Näke, C., Blazevic, D., Grefen, C., Schumacher, K., Oecking, C., Harter, K., and Kudla, J. 2004. Visualization of protein interactions in living plant cells using bimolecular fluorescence complementation. Plant J. 40: 428-438.

Wang, J., Sykes, B. D., and Ryan, R. O. 2002. Structural basis for the conformational adaptability of apolipophorin III, a helix-bundle exchangeable apolipoprotein. Proc. Natl. Acad. Sci. U.S.A. 99: 1188-1193.

Wang, Q., Han, C., Ferreira, A. O., Yu, X., Ye, W., Tripathy, S., Kale, S. D., Gu, B., Sheng, Y., Sui, Y., Wang, X., Zhang, Z., Cheng, B., Dong, S., Shan, W., Zheng, X., Dou, D., Tyler, B. M., and Wang, Y. 2011. Transcriptional programming and functional interactions within the Phytophthora sojae RXLR effector repertoire. Plant Cell 23: 2064-2086.

Wang, W., Dai, H., Zhang, Y., Chandrasekar, R., Luo, L., Hiromasa, Y., Sheng, C., Peng, G., Chen, S., Tomich, J. M., Reese, J., Edwards, O., Kang, L., Reeck, G., and Cui, F. 2015a. Armet is an effector protein mediating aphid-plant interactions. FASEB J. 29:2032-2045.

Wang, W., Luo, L., Lu, H., Chen, S., Kang, L., and Cui, F. 2015b. Angiotensin-converting enzymes modulate aphid-plant interactions. Sci. Rep. 5: Article 8885.

Wang, Y., Tang, M., Hao, P., Yang, Z., Zhu, L., and He, G. 2008. Penetration into rice tissues by brown planthopper and fine structure of the salivary sheaths. Entomol. Exp. Appl. 129:295-307.

Wroblewski, T., Caldwell, K. S., Piskurewicz, U., Cavanaugh, K. A., Xu, H., Kozik, A., Ochoa, O., McHale, L. K., Lahre, K., Jelenska, J., Castillo, J. A., Blumenthal, D., Vinatzer, B. A., Greenberg, J. T., and Michelmore, R. W. 2009. Comparative large-scale analysis of interactions between several crop species and the effector repertoires from multiple pathovars of Pseudomonas and Ralstonia. Plant Physiol. 150:1733-1749.

Xue, J., Zhou, X., Zhang, C. X., Yu, L. L., Fan, H. W., Wang, Z., Xu, H. J., Xi, Y., Zhu, Z. R., Zhou, W. W., Pan, P. L., Li, B. L., Colbourne, J. K., Noda, H., Suetsugu, Y., Kobayashi, T., Zheng, Y., Liu, S., Zhang, R., Liu, Y., Luo, Y. D., Fang, D. M., Chen, Y., Zhan, D. L., Lv, X. D., Cai, Y., Wang, Z. B., Huang, H. J., Cheng, R. L., Zhang, X. C., Lou, Y. H., Yu, B., Zhuo, J. C., Ye, Y. X., Zhang, W. Q., Shen, Z. C., Yang, H. M., Wang, J., Wang, J., Bao, Y. Y., and Cheng, J. A. 2014. Genomes of the rice pest brown planthopper and its endosymbionts reveal complex complementary contributions for host adaptation. Genome Biol. 15:521.

Ye, W., Yu, H., Jian, Y., Zeng, J., Ji, R., Chen, H., and Lou, Y. 2017. A salivary EF-hand calcium-binding protein of the brown planthopper Nilaparvata lugens functions as an effector for defense responses in rice. Sci. Rep. 7: Article 40498.

Zhang, Y., Fan, J., Sun, J., and Chen, J. 2015. Cloning and RNA interference analysis of the salivary protein $\mathrm{C} 002$ gene in Schizaphis graminum. J. Integr. Agric. 14:698-705.

Zhou, W., Yuan, X., Qian, P., Cheng, J., Zhang, C., Gurr, G., and Zhu, Z. 2015. Identification and expression profiling of putative chemosensory protein genes in two rice planthoppers, Laodelphax striatellus (Fallén) and Sogatella furcifera (Horváth). J. Asia Pac. Entomol. 18:771-778. 